TRANSACTIONS OF THE

AMERICAN MATHEMATICAL SOCIETY

Volume 363, Number 9, September 2011, Pages 4735-4755

S 0002-9947(2011)05203-7

Article electronically published on April 8, 2011

\title{
WEAK EXPECTATIONS AND THE INJECTIVE ENVELOPE
}

\author{
VERN I. PAULSEN
}

\begin{abstract}
Given a unital $C^{*}$-subalgebra $\mathcal{A} \subseteq B(\mathcal{H})$, we study the set of all possible images of the injective envelope $I(\mathcal{A})$ of $\mathcal{A}$ that are contained in $B(\mathcal{H})$ and their position relative to the double commutant of the algebra in order to develop more information about the existence or non-existence of weak expectations. We study the set of all elements of $B(\mathcal{H})$ that are fixed by all completely positive maps that fix $\mathcal{A}$. We also introduce a new category, such that the injective envelope of $\mathcal{A}$ in the new category is always contained in the double commutant of $\mathcal{A}$. We study the relationship between these two injective envelopes and the existence of weak expectations.
\end{abstract}

\section{INTRODUCTION AND PRELIMINARIES}

A unital $C^{*}$-subalgebra $\mathcal{A} \subseteq B(\mathcal{H})$ of the bounded linear operators on a Hilbert space is said to have a weak expectation provided that there is a completely positive map from $B(\mathcal{H})$ into the double commutant of $\mathcal{A}$, denoted $\mathcal{A}^{\prime \prime}$, that is the identity on $\mathcal{A}$. A unital $C^{*}$-algebra $\mathcal{A}$ is said to have the weak expectation property (WEP) provided that for every faithful *-representation $\pi: \mathcal{A} \rightarrow B(\mathcal{H})$ of $\mathcal{A}$ onto a Hilbert space $\mathcal{H}$, the $C^{*}$-subalgebra $\pi(\mathcal{A})$ has a weak expectation. If we let $\pi_{u}$ denote the universal representation of $\mathcal{A}$, so that the double commutant $\pi_{u}(A)^{\prime \prime}$ is identified with the double dual, $A^{* *}$, then it is known that $\mathcal{A}$ has the WEP if and only if this representation has a weak expectation.

Blackadar 2] observed that $\mathcal{A}$ has a weak expectation if and only if $\mathcal{A}^{\prime \prime}$ contains an operator system that is completely isometrically isomorphic to $I(\mathcal{A})$, the injective envelope of $\mathcal{A}$, via a map that fixes $\mathcal{A}$. Thus, the WEP is equivalent to the existence of a copy of $I(\mathcal{A})$ inside $\mathcal{A}^{* *}$.

However, in general, $B(\mathcal{H})$ will contain many operator systems that contain $\mathcal{A}$ and are completely isometrically isomorphic to $I(\mathcal{A})$. For a weak expectation to exist we only need one of these copies of the injective envelope to be contained in the double commutant. In general, a $C^{*}$-subalgebra $\mathcal{A} \subseteq B(\mathcal{H})$, which has a weak expectation, will also have some copies of $I(\mathcal{A})$ that are not contained in $\mathcal{A}^{\prime \prime}$. We construct such an algebra below.

For this reason, given a unital $C^{*}$-subalgebra $\mathcal{A} \subseteq B(\mathcal{H})$, we are led to a more detailed study of the collection of all possible "copies" of $I(\mathcal{A})$ that lie inside $B(\mathcal{H})$,

Received by the editors July 24, 2008 and, in revised form, August 25, 2009 and September 1, 2009.

2010 Mathematics Subject Classification. Primary 46L07; Secondary 47L25.

Key words and phrases. Weak expectation, injective.

This research was supported in part by NSF grant DMS-0600191.

(C)2011 American Mathematical Society 
the relationships between these various copies of the injective envelopes and the collection of projections onto these copies. One object that will play a role in our study is the intersection of all possible copies of $I(\mathcal{A})$.

Another tool that we shall use is a new type of "envelope" of $\mathcal{A}$ that is always a subset of $\mathcal{A}^{\prime \prime}$, but which is, generally, only injective in a sense relative to $\mathcal{A}^{\prime \prime}$. It turns out that $\mathcal{A}$ has a weak expectation if and only if this new envelope is injective in the usual sense.

In section 2, we further develop some of Hamana's ideas. We introduce and study this new type of envelope and simultaneously obtain additional information about the set of all projections onto copies of the injective envelope. This is the set that Hamana [6] calls the minimal $\mathcal{A}$-projections. Section 3 applies these ideas to the study of weak expectations. Section 4 is devoted to developing the properties of the set that is the intersection of all copies of the injective envelope. We prove that this set is simultaneously a reflexive cover of $\mathcal{A}$ and a new type of order completion of $\mathcal{A}$. We compute this set for a few examples.

We close this section by justifying a few of the comments above. First, since the entire motivation for this study relies on Blackadar's result [2], we provide an independent argument which also serves as an introduction to many of Hamana's ideas.

To obtain Blackadar's result, first assume that $\mathcal{A} \subseteq B(\mathcal{H})$ possesses a weak expectation. Note that since $B(\mathcal{H})$ is injective, the identity map on $\mathcal{A}$ extends to a map of $I(\mathcal{A})$ into $B(\mathcal{H})$. Composing this latter map with the weak expectation yields a completely positive map of $I(\mathcal{A})$ into $\mathcal{A}^{\prime \prime}$ that fixes $\mathcal{A}$. The map of $I(\mathcal{A})$ into $\mathcal{A}^{\prime \prime}$ must be a complete isometry on the injective envelope, by the fact [6] that the injective envelope is an essential extension of $\mathcal{A}$. Conversely, if $I(\mathcal{A})$ can be embedded completely isometrically isomorphically into $\mathcal{A}^{\prime \prime}$, then since $I(\mathcal{A})$ is injective, we may extend the identity map on $\mathcal{A}$ to a completely positive map of $B(\mathcal{H})$ to $I(\mathcal{A})$. Composing this extension with the inclusion of $I(\mathcal{A})$ into $\mathcal{A}^{\prime \prime}$ yields the desired weak expectation.

Next we would like to point out that the fact that the image of one representation $\pi: \mathcal{A} \rightarrow B(\mathcal{H})$ has a weak expectation is not enough to guarantee that $\mathcal{A}$ has the weak expectation property. In fact, every $C^{*}$-algebra has at least one representation that has a weak expectation. The double commutant of the reduced atomic representation is always injective, and hence this representation possesses a weak expectation.

Next we give an example to show that it is possible for a $C^{*}$-algebra to have a weak expectation, while some copies of the injective envelope are not contained inside the double commutant of the algebra. To this end, let $\mathcal{A}$ be a $C^{*}$-algebra with the WEP that is not injective and take the universal representation of the $C^{*}$-algebra, $I(\mathcal{A})$. Then $\pi_{u}(I(\mathcal{A}))$ is a copy of the injective envelope of $\pi_{u}(\mathcal{A})$, but we claim that this copy of the injective envelope is not contained in $\pi_{u}(\mathcal{A})^{\prime \prime}$. If not, then we would have that $\pi_{u}(I(\mathcal{A})) \subseteq \pi_{u}(\mathcal{A})^{\prime \prime}$. But this implies that $I(\mathcal{A})^{* *} \simeq$ $\pi_{u}(I(\mathcal{A}))^{\prime \prime} \subseteq \pi_{u}(\mathcal{A})^{\prime \prime} \simeq \mathcal{A}^{* *}$ and this inclusion of $I(\mathcal{A})^{* *} \subseteq \mathcal{A}^{* *}$ is weak*-continuous and the identity on $\mathcal{A}$. From this it follows that $\mathcal{A}=I(\mathcal{A})$, contradicting the choice of $\mathcal{A}$. Since $\mathcal{A}$ had the WEP, $\pi_{u}(\mathcal{A})$ is a $C^{*}$-algebra with a weak expectation and so some copy of its injective envelope is contained in $\pi_{u}(\mathcal{A})^{\prime \prime}$, but $\pi_{u}(I(\mathcal{A})) \simeq I\left(\pi_{u}(\mathcal{A})\right.$ ) is a copy of its injective envelope that is not contained in the double commutant.

Finally, although our main interest is in the case of $C^{*}$-algebras, many of the ideas extend to the case of operator spaces. 
The definition of the injective envelope of a $C^{*}$-algebra has been extended to general operator spaces by Ruan [1] and has been shown to enjoy similar properties to those proven by Hamana, namely, that it is a rigid, essential and injective extension. Pisier 10] defined an operator space $V$ to have the WEP provided that the identity on $V$ extends to a completely contractive map of Ruan's injective envelope $I(V)$ into $V^{* *}$. Thus, a finite dimensional operator space has the WEP if and only if it is injective. We prove below that Pisier's definition, as in the case of $C^{*}$-algebras, is equivalent to one involving weak expectations.

Definition 1.1. Let $V \subseteq B(\mathcal{H})$ be an operator space. We say that $V$ has a weak expectation if there is a completely contractive map from $B(\mathcal{H})$ into the weak*closure of $V$, that is the identity on $V$. We define an abstract operator space $V$ to have the weak expectation property (WEP) provided that there exists a completely isometric embedding of the injective envelope of $V, I(V)$ into $V^{* *}$ that is the identity on $V$.

The equivalence of the WEP to every inclusion possessing a weak expectation is a little more subtle for operator spaces, so we prove this below.

Proposition 1.2. Let $V$ be an operator space. Then $V$ has the WEP if and only if for every Hilbert space, $\mathcal{H}$, and for every complete isometry, $\varphi: V \rightarrow B(\mathcal{H})$, the subspace $\varphi(V) \subseteq B(\mathcal{H})$ has a weak expectation.

Proof. Assume that $V$ has the WEP and let $\varphi: V \rightarrow B(\mathcal{H})$ be a complete isometry. Let $W$ denote the weak*-closure of $\varphi(V)$, so that by [3, Lemmas 1.4.6 and 1.4.8] $W$ has a pre-dual and $\varphi$ extends to a complete contraction of $V^{* *}$ into $W$. Composing this map with the embedding of $I(V)$ into $V^{* *}$ yields a completely contractive mapping of $I(V)$ into $W$ that restricts to a complete isometry on $V$. Since $I(V)$ is an essential extension of $V$, this map must also be a complete isometry on $I(V)$.

Conversely, assume that every completely isometric embedding of $V$ into $B(\mathcal{H})$ possesses a weak expectation. By [3. Lemma 1.4.7], there exists a weak*-continuous completely isometric embedding of $V^{* *}$ onto a weak*-closed subspace of $B(\mathcal{H})$, for some $\mathcal{H}$. Taking any weak expectation for this embedding and composing it with any completely isometric inclusion of $I(V)$ into $B(\mathcal{H})$, yields the desired embedding of $I(V)$ into $V^{* *}$.

We remark that the above proof applied to the case of $C^{*}$-algebras shows the earlier equivalence of the two characterizations of WEP.

\section{Minimal PRojections AND $W$-InJECTIVITY}

In this section we take a closer look at the ideas contained in Hamana's constructions 6, 7 of the injective envelope and prove a number of facts that are consequences of these ideas, but that seem to have not been observed earlier. In order to better understand the weak expectations it is useful to examine the extent that Hamana's constructions can be carried out in the setting where one has operator spaces $V \subseteq W$ with $W$ a dual operator space that is not necessarily injective.

Definition 2.1. Let $W$ be an operator space. We let $\mathfrak{W}$ denote the category whose objects are operator subspaces of $W$ and given two operator subspaces $X, Y$ of $W$ we let the maps from $X$ to $Y$, denoted by $M(X, Y)$, be the set of completely contractive maps from $\phi: W \rightarrow W$ such that $\phi(X) \subseteq Y$. We define an object $Y$ to 
be injective in $\mathfrak{W}$ or more shortly, $W$-injective provided that whenever $X_{1} \subset X_{2}$ are operator subspaces of $W$ and $\phi_{1} \in M\left(X_{1}, Y\right)$, then there exists $\phi_{2} \in M\left(X_{2}, Y\right)$ such that the restriction of $\phi_{2}$ to $X_{1}$ is $\phi_{1}$. We say that $X$ and $Y$ are $W$-isomorphic provided that there exists $\phi \in M(X, Y)$ and $\psi \in M(Y, X)$ such that $\psi \circ \phi$ is the identity on $X$ and $\phi \circ \psi$ is the identity on $Y$.

It is not hard to see that $Y$ is $W$-injective if and only if there exists a completely contractive idempotent from $W$ onto $Y$. So, for example, $W$ is always injective in $\mathfrak{W}$, even though it need not be an injective operator space in the traditional sense. If $W$ is an injective operator space in the usual sense, then it follows that every $W$-injective operator space is also injective in the usual sense. If $X$ and $Y$ are $W$-isomorphic, then they are completely isometrically isomorphic, but the converse is not apparent.

We now show that when $W$ is a dual space, then many of Hamana's results about minimal projections and injective envelopes hold and allow one to construct an injective envelope in $\mathfrak{W}$ with analogous properties to the usual injective envelope.

We begin with the relevant definitions.

Definition 2.2. Let $V \subseteq W$ be operator spaces. We call a completely contractive map $\phi: W \rightarrow W$ a $V$-map provided that $\phi(v)=v$ for every $v \in V$. We say that $X$ is a $W$-essential extension of $V$ provided that $V \subseteq X \subseteq W$ and whenever $\phi: W \rightarrow W$ is a $V$-map, then there exists a $V$-map $\psi$ such that $\psi \circ \phi(x)=x$ for every $x \in X$. We say that $X$ is a $W$-rigid extension of $V$ provided that $V \subseteq X \subseteq W$ and the only $V$-map in $M(X, X)$ is the identity map on $X$.

When $W$ is injective in the usual sense, then $X$ is a $W$-rigid (respectively, $W$ essential) extension of $V$ if and only if it is a rigid (respectively, essential) extension of $V$ in the usual sense, that is, if and only if the only completely contractive map from $X$ to itself that fixes $V$ is the identity on $X$ (respectively, any completely contractive map on $X$ that is a complete isometry on $V$ is a complete isometry on $X)$.

Given a $V$-map $\phi: W \rightarrow W$ it induces a seminorm on $W$ by setting $p_{\phi}(w)=$ $\|\phi(w)\|$. The set of such seminorms is partially ordered by $p_{\phi} \leq p_{\psi}$ if and only if $p_{\phi}(w) \leq p_{\psi}(w)$ for every $w \in W$.

Now assume that $W$ is a dual Banach space, so that $W$ is endowed with a weak*topology. Given any chain of such seminorms, then by taking a point-weak*-limit point of the set of maps, we obtain a new $V$-map $\psi$ such that $p_{\psi}$ is a lower bound for the chain. Thus, by Zorn's lemma, there exist minimal such seminorms.

To use the above argument to prove the existence of minimal seminorms, one only needs that $M(W, W)$ is endowed with a topology that makes it a compact set and such that for every $w \in W$ the map $\phi \rightarrow\|\phi(w)\|$ is lower semicontinuous.

Definition 2.3. We call an operator space $W$ admissible provided that for every operator subspace $V$ and every $V$-map $\phi$ there exists a $V$-map $\psi$ such that $p_{\psi} \leq p_{\phi}$ with $p_{\psi}$ a minimal seminorm.

Thus, by our above remarks, every operator space $W$ that has an appropriate topology on $M(W, W)$ is admissible and in particular every operator space that is a dual Banach space is admissable.

Theorem 2.4. Let $V \subseteq W$ be operator spaces with $W$ admissible and let $\phi$ : $W \rightarrow W$ be a $V$-map such that the seminorm $p_{\phi}$ is minimal among this family of 
seminorms. Then $\phi$ is a completely contractive projection and the range of $\phi, \mathcal{R}(\phi)$ is a $W$-rigid, $W$-injective extension of $V$.

Proof. Set $\phi^{(2)}=\phi \circ \phi$ and inductively, $\phi^{(n+1)}=\phi \circ \phi^{(n)}$. Define $\psi_{n}=\frac{\phi+\cdots+\phi^{(n)}}{n}$. Since $p_{\psi_{n}} \leq p_{\phi}$ we have equality of these seminorms. But

$$
\psi_{n}(x-\phi(x))=\frac{\phi(x)-\phi^{(n+1)}(x)}{n},
$$

which tends to 0 in norm. Hence, $0=\phi(x-\phi(x))$ and so it follows that $\phi$ is idempotent and so the range of $\phi$ is $W$-injective.

Letting $Y=\mathcal{R}(\phi)$, it remains to show that $Y$ is a $W$-rigid extension of $V$. Let $\psi \in M(Y, Y)$ be a $V$-map. Since $p_{\psi \circ \phi} \leq p_{\phi}$ we again have equality. Thus, by the above, $\psi \circ \phi$ must be an idempotent map. Let $y \in Y$. Then

$$
\|y-\psi(y)\|=\|\phi(y-\psi \circ \phi(y))\|=\| \psi \circ \phi(y-\psi \circ \phi(y) \|=0 .
$$

Hence, $\psi$ is the identity on $Y$ and so $Y$ is a $W$-rigid extension of $V$.

For all of the following results, we assume that $W$ is an admissible operator space and that $V \subset W$.

Lemma 2.5. Let $W$ be an admissible operator space, with $V \subset W$. Let $Y$ be a $W$-rigid and $W$-injective extension of $V$. Let $E: W \rightarrow Y$ be $a V$-map and let $\psi$ be any $V$-map. If $p_{\psi} \leq p_{E}$, then $E \circ \psi=E$ and $\operatorname{ker}(\psi)=\operatorname{ker}(E)$.

Proof. Since $Y$ is a $W$-rigid extension of $V$, we have that $E(y)=y$ for every $y \in Y$, and hence, $E \circ E=E$. For $y \in Y$ we have that $E \circ \psi(y)=y$ by rigidity. For $k \in \operatorname{ker}(E)$ we have that $\|\psi(k)\| \leq\|E(k)\|=0$ and so $\operatorname{ker}(E) \subseteq \operatorname{ker}(\psi)$. Since every element $x \in W$ can be written as $x=y+k$ for $y \in Y$ and $k \in \operatorname{ker}(E)$, we have that $E \circ \psi(x)=y=E(x)$ and the first claim follows.

If $x \in \operatorname{ker}(\psi)$, then $E(x)=E \circ \psi(x)=0$ and so $\operatorname{ker}(\psi) \subseteq \operatorname{ker}(E)$.

Proposition 2.6. Let $Y$ be a $W$-rigid and $W$-injective extension of $V$ and let $E: W \rightarrow Y$ be a $V$-map. Then $p_{E}$ is a minimal $V$-seminorm.

Proof. Suppose not. Then we may choose a $V$-map $\phi$ such that $p_{\phi} \leq p_{E}$ with $\|\phi(x)\|<\|E(x)\|$ for some $x$. But by the above, $\|E(x)\|=\|E \circ \phi(x)\| \leq\|\phi(x)\|$, a contradiction.

Proposition 2.7. Let $V \subseteq W$ with $W$ admissible and let $Y$ be a $W$-rigid and $W$-injective extension of $V$. Let $E: W \rightarrow Y$ be a $V$-map and let $\phi$ be any $V$-map. Then $\operatorname{ker}(\phi \circ E)=\operatorname{ker}(E), E \circ \phi \circ E=E$ and $\phi \circ E$ and $E \circ \phi$ are completely contractive projections onto $W$-rigid and $W$-injective extensions of $V$.

Proof. Since $\phi$ is a contraction, $p_{\phi \circ E} \leq p_{E}$ and so we may apply Lemma 2.5 with $\phi \circ E=\psi$ to obtain that $E \circ \phi \circ E=E$ and that $\operatorname{ker}(\phi \circ E)=\operatorname{ker}(E)$. Hence, $(\phi \circ E) \circ(\phi \circ E)=\phi \circ E$ and $(E \circ \phi) \circ(E \circ \phi)=E \circ \phi$ and so these maps are completely contractive projections, as claimed. Taking $\phi=i d_{W}$ we have that $E$ is also a completely contractive projection.

It remains to show that $\mathcal{R}(\phi \circ E)$ and $\mathcal{R}(E \circ \phi)$ are $W$-rigid, $W$-injective extensions of $V$. By Proposition 2.6, $p_{E}$ is a minimal $V$-seminorm and hence $p_{\phi \circ E}$ is also a minimal $V$-seminorm and hence by Theorem 2.4, $\mathcal{R}(\phi \circ E)$ is a $W$-rigid and $W$ injective extension of $V$. Also, since $E \circ \phi \circ E=E$, we have that $\mathcal{R}(E \circ \phi)=$ $\mathcal{R}(E)=Y$, which is a $W$-rigid and $W$-injective extension of $V$. 
Corollary 2.8. Let $V \subseteq W$ with $W$ admissible and let $\phi: W \rightarrow W$ be a $V$-map whose range is contained in a $W$-injective, $W$-rigid extension $Y$ of $V$. Then $\phi$ is a projection onto $Y$.

Proof. Let $E$ be a projection onto $Y$. Then $E \circ \phi=\phi$. By the above result $E \circ \phi$ is a projection onto a $W$-injective subspace of $Y$ which, by the $W$-rigidity of $Y$, must be all of $Y$.

Recall that Hamana [6] introduces a partial order on projections by defining $E \preceq F$ if and only if $E \circ F=F \circ E=E$. Note that this is equivalent to requiring that $E \circ F \circ E=F \circ E \circ F=E$. To see this note that the first set of equalities clearly implies the second set. If the second set of equalities holds, then $F \circ E=$ $F \circ(E \circ F \circ E)=(F \circ E \circ F) \circ E=E^{2}=E$ and similarly, $E \circ F=E$.

Theorem 2.9. Let $V \subseteq W$ with $W$ admissible and let $E: W \rightarrow W$ be a $V$-map. Then the following are equivalent:

i) $p_{E}$ is a minimal $V$-seminorm,

ii) $E$ is a projection onto a $W$-injective, $W$-rigid extension of $V$,

iii) $E$ is minimal in the partial order on $V$-projections.

Moreover, if $E_{1}, E_{2}$ are two such minimal $V$-projections, then $\mathcal{R}\left(E_{1}\right)$ and $\mathcal{R}\left(E_{2}\right)$ are $W$-isomorphic.

Proof. The proof that i) implies ii) is Theorem 2.4 and that ii) implies i) is Proposition 2.6. Assume ii) and let $F \preceq E$. Then, by Corollary 2.8, $F$ is also a projection onto $Y$. Hence, $E=F \circ E=F$ and so $E$ is minimal.

Next, assuming iii), let $p_{F} \leq p_{E}$ be a minimal $V$-seminorm. Then $p_{E \circ F}$ is also a minimal $V$-seminorm and so $F$ and $E \circ F$ are projections onto $W$-injective, $W$ rigid extensions of $V$. Hence by another application of Corollary 2.8, $E \circ F \circ E$ is another projection onto a $W$-injective, $W$-rigid extension of $V$. But $E \circ(E \circ F \circ E)=$ $E \circ F \circ E=(E \circ F \circ E) \circ E$ and hence $E=E \circ F \circ E$. Thus, $E$ is a projection onto a $W$-injective, $W$-rigid extension of $V$.

Finally, if $E_{1}$ and $E_{2}$ are minimal $V$-projections, with ranges $Y_{1}$ and $Y_{2}$, respectively, then $E_{2} \circ E_{1}$ defines a completely isometric $W$-isomorphism of $Y_{1}$ onto $Y_{2}$, with inverse $E_{1} \circ E_{2}$.

Thus, we see that all $W$-injective, $W$-rigid extensions of $V$ are $W$-isomorphic, provided $W$ is admissible.

Definition 2.10. Let $W$ be an admissible operator space and $V$ a subspace. We call any $V$-map $E: W \rightarrow W$ that satisfies the equivalent properties of Theorem 2.9 a minimal $V$-projection (with respect to $W$ ) and let $\mathcal{E}_{W}(V)$ denote the set of all minimal $V$-projections. We let $I_{W}(V)$ denote the $W$-isomorphism class of the range of a minimal $V$-projection and we call this operator space the $W$-injective envelope of $V$. Any operator subspace of $W$ that is the range of a minimal $V$-projection will be called a copy of $I_{W}(V)$.

When $V=\mathcal{A}$ is a unital $C^{*}$-algebra and $W=\mathcal{A}^{\prime \prime}$, then any minimal $\mathcal{A}$-projection $E$ is also a unital, completely positive map and, hence, $I_{\mathcal{A}^{\prime \prime}}(\mathcal{A})$ will be an $\mathcal{A}^{\prime \prime}$ injective $C^{*}$-algebra when endowed with the Choi-Effros product, $E(x) \circ E(y)=$ $E(E(x) E(y))$.

When $W$ is injective, then $I_{W}(V)=I(V)$, the usual injective envelope. 
The following result shows that, in general, $\mathcal{E}_{W}(V)$ can be quite large and explains some of its algebraic structure. Note that the set of $V$-maps, which we shall denote by $\mathcal{M}_{W}(V)$, is a semigroup under composition with the identity map on $W$ serving as an identity.

Proposition 2.11. Let $W$ be an admissible operator space and let $V$ be an operator subspace. Then $\mathcal{E}_{W}(V)$ is the unique minimal, non-empty, two-sided ideal in the semigroup $\mathcal{M}_{W}(V)$.

Proof. Given any $V$-map $\phi$ and $E \in \mathcal{E}_{W}(V)$, we have that $\phi \circ E \in \mathcal{E}_{W}(V)$ and $E \circ \phi \in \mathcal{E}_{W}(V)$, by Proposition 2.7 and Theorem 2.9. Thus, $\mathcal{E}_{W}(V)$ is a two-sided ideal in $\mathcal{M}_{W}(V)$.

Given any non-empty two-sided ideal $\mathcal{J}$ in $\mathcal{M}_{W}(V)$, let $\phi \in \mathcal{J}$, and let $E \in$ $\mathcal{E}_{W}(V)$. Then by Proposition 2.7, $E=E \circ \phi \circ E \in \mathcal{J}$, and hence, $\mathcal{E}_{W}(V) \subseteq \mathcal{J}$.

The following result identifies the minimal left ideals. Given any $\phi \in \mathcal{M}_{W}(V)$, we let $\mathcal{L}_{\phi}=\left\{\psi \circ \phi: \psi \in \mathcal{M}_{W}(V)\right\}$ denote the left ideal generated by $\phi$.

Theorem 2.12. Let $W$ be an admissible operator space, let $V$ be an operator subspace, and let $E \in \mathcal{E}_{W}(V)$. Then $\mathcal{L}_{E}=\left\{F \in \mathcal{E}_{W}(V): p_{F}=p_{E}\right\}=\{F \in$ $\left.\mathcal{E}_{W}(V): \operatorname{ker}(F)=\operatorname{ker}(E)\right\}, \mathcal{L}_{E}$ is a convex set and a minimal non-empty left ideal in the semigroup of $V$-maps. Moreover, every minimal, non-empty left ideal in $\mathcal{M}_{W}(V)$ is equal to $\mathcal{L}_{E}$ for some $E \in \mathcal{E}_{W}(V)$. Finally, if $F_{1}, F_{2} \in \mathcal{L}_{E}$, then $F_{1}+F_{2}=F_{1} \circ F_{2}+F_{2} \circ F_{1}$.

Proof. First we show that the three sets are equal. If $\phi \circ E \in \mathcal{L}_{E}$, then $\|\phi \circ E(w)\| \leq$ $\|E(w)\|$, so by minimality, $p_{\phi \circ E}=p_{E}$, so the first set is contained in the second. If $p_{F}=p_{E}$, then, clearly, $\operatorname{ker}(F)=\operatorname{ker}(E)$, so the second set is contained in the third. If $\operatorname{ker}(F)=\operatorname{ker}(E)$, then since $E(w-E(w))=0$, we have that $F(w)=F \circ E(w)$, so that $F=F \circ E \in \mathcal{L}_{E}$, and all three sets are equal.

Let $F_{1}, F_{2} \in \mathcal{L}_{E}$ and let $0 \leq t \leq 1$. Then for any $w \in W$, we have that $\left\|t F_{1}(w)+(1-t) F_{2}(w)\right\| \leq\|E(w)\|$. Since $p_{E}$ is a minimal $V$-seminorm, it follows that $p_{t F_{1}+(1-t) F_{2}}=p_{E}$ and hence, $t F_{1}+(1-t) F_{2} \in \mathcal{L}_{E}$. Thus, $\mathcal{L}_{E}$ is convex.

Now, let $\mathcal{J} \subseteq \mathcal{L}_{E}$ be any non-empty left ideal, and let $\phi \in \mathcal{J}$, so that $E \circ \phi \in \mathcal{J}$. Since $\phi \in \mathcal{L}_{E}$, there exists $\psi \in \mathcal{M}_{W}(V)$ such that $\phi=\psi \circ E$ and, hence, $E \circ \phi=$ $E \circ \psi \circ E=E$ by Proposition 2.7. Thus, $E \in \mathcal{J}$ and so, $\mathcal{L}_{E} \subseteq \mathcal{J}$. This proves that $\mathcal{L}_{E}$ is a minimal, non-empty left ideal.

Finally, since $\left(F_{1}+F_{2}\right) / 2 \in \mathcal{L}_{E}$, we have that $\left(F_{1}+F_{2}\right) / 2=\left(F_{1}+F_{2}\right) / 2 \circ\left(F_{1}+\right.$ $\left.F_{2}\right) / 2=1 / 4\left(F_{1}+F_{1} \circ F_{2}+F_{2} \circ F_{1}+F_{2}\right)$, which implies $F_{1}+F_{2}=F_{1} \circ F_{2}+F_{2} \circ F_{1}$.

Corollary 2.13. Let $W$ be an admissible operator space and let $V$ be a subspace. Then $\mathcal{E}_{W}(V)$ is the disjoint union of the minimal left ideals in $\mathcal{M}_{W}(V)$.

Proof. Every minimal non-empty left ideal is of the form $\mathcal{L}_{E}$ for some $E \in \mathcal{E}_{W}(V)$, and $\mathcal{L}_{E} \subseteq \mathcal{M}_{W}(V)$ and any two such ideals are either disjoint or equal.

Similar results hold for the right ideal, $\mathcal{R}_{E}$, generated by $E \in \mathcal{E}_{W}(V)$. We record some of them without proof.

Proposition 2.14. Let $W$ be an admissible operator space, let $V$ be an operator subspace and let $E \in \mathcal{E}_{W}(V)$. Then $\mathcal{R}_{E}=\left\{F \in \mathcal{E}_{W}(V): \mathcal{R}(F)=\mathcal{R}(E)\right\}$ and $\mathcal{R}_{E}$ is a convex set and a minimal right ideal. Moreover, every minimal, non-empty right ideal is equal to $\mathcal{R}_{E}$ for some $E \in \mathcal{E}_{W}(V)$ and $\mathcal{E}_{W}(V)$ is the disjoint union of all minimal right ideals. 
The following result gives a way to obtain copies of $I_{W}(V)$ and clarifies the relationship of $I_{W}(V)$ with the usual injective envelope.

Theorem 2.15. Let $V \subseteq W \subseteq B(\mathcal{H})$ with $W$ admissible and let $\phi: W \rightarrow W$ be a minimal $V$-projection, so that $Y=\mathcal{R}(\phi)$ is a copy of $I_{W}(V)$. Then there is a copy $S \subseteq B(\mathcal{H})$ of $I(Y)$ and a completely contractive projection $E: B(\mathcal{H}) \rightarrow S$ that is an extension of $\phi$ to $B(\mathcal{H})$ such that $Y=W \cap S$.

Proof. Among all extensions of $\phi$ to $B(\mathcal{H})$ choose one, say $E$, such that the induced seminorm on $B(\mathcal{H})$ is minimal among the set of all such seminorms. The existence of such a minimal seminorm is guaranteed by Zorn's lemma, since every chain has a lower bound given by taking a point weak*-limit point, as above. Setting $\psi_{n}=\frac{E+E \circ E+\ldots+E^{(n)}}{n}$, we have that $\psi_{n}$ still extends $\phi$ and produces a smaller seminorm on $B(\mathcal{H})$ and, consequently, must be equal to the seminorm induced by $E$. Apply to $x-E(x)$, as before, to deduce that $E$ is idempotent.

Hence $E$ is a projection onto some (necessarily) injective operator space $S$ and from this it follows that $\phi$ is the projection onto $W \cap S$.

We now prove that $S$ is a rigid extension of $Y$. To this end suppose that $\gamma: S \rightarrow S$ is a completely contractive map that fixes $Y$. Then $\gamma \circ E$ is another extension of $\phi$ with $p_{\gamma \circ E} \leq p_{E}$ and hence we must have equality of these two seminorms. Hence $\gamma \circ E$ must also be idempotent. Arguing as in the last line of Theorem 2.4 we obtain that $\|s-\gamma(s)\|=0$ and so $\gamma$ is the identity on $S$.

Since $S$ is injective and a rigid extension of $Y$ we have that $S$ is completely isometrically isomorphic to $I(Y)$ via a map that fixes $Y$. That is, $S$ is one of the copies of $I(Y)$ in $B(\mathcal{H})$.

If $S$ is an arbitrary copy of $I(Y)$ in $B(\mathcal{H})$, then it might not be the case that $W \cap S=Y$ or that the projection $E$ onto $S$ satisfies $E(W) \subseteq W$, but we do not have a concrete example where these fail.

Note that since $V \subseteq Y$, we will have that any copy of $I(Y)$ will contain a copy of $I(V)$. Moreover, since any two copies $Y_{1}, Y_{2}$ of $I_{W}(V)$ are $W$-isomorphic, they are completely isometrically isomorphic via a map that fixes $V$ and hence, any copies of $I\left(Y_{1}\right)$ and $I\left(Y_{2}\right)$ in $B(\mathcal{H})$ will be completely isometrically isomorphic via a map that fixes $V$. But we do not know if $I(V)=I(Y)$, or equivalently, if any completely contractive map $\psi: I(Y) \rightarrow I(Y)$ that fixes $V$ is necessarily the identity map.

Definition 2.16. Let $V \subseteq W$ with $W$ admissible. If $V \subseteq Y \subseteq W$ is any copy of $I_{W}(V)$, then we set $I^{W}(V)=I(Y)$ and recall that this operator space is uniquely determined up to a completely isometric isomorphism that fixes $V$ and is independent of $Y$.

The following gives a characterization of $I^{W}(V)$ in the main case of interest.

Proposition 2.17. Let $V \subseteq W \subseteq B(\mathcal{H})$, with $W$ admissible and let $E: B(\mathcal{H}) \rightarrow$ $B(\mathcal{H})$ be a $V$-map. If $p_{E}$ is minimal among all $V$-seminorms on $B(\mathcal{H})$ such that $E(W) \subseteq W$, then $E(B(\mathcal{H}))$ is a copy of $I^{W}(V)$ and $E(W)$ is a copy of $I_{W}(V)$.

Proof. If $p_{E}$ is minimal in the above sense, then arguing as in the proof of Theorem 2.4, we see that $E$ is idempotent and hence a $V$-projection. Let $\phi: W \rightarrow W$ be the restriction of $E$ to $W$.

We claim that $p_{\phi}$ is a minimal $V$-seminorm on $W$. If not, then we have that $\psi: W \rightarrow W$ is a $V$-map such that $p_{\psi} \leq p_{\phi}$. Then we have that $\|\psi(x-\phi(x))\| \leq$ $\|\phi(x-\phi(x))\|=0$, and hence, $\psi(x)=\psi \circ \phi(x)$, for any $x \in W$. 
Let $F: B(\mathcal{H}) \rightarrow B(\mathcal{H})$ be any completely contractive extension of $\psi$. Then $p_{F \circ E} \leq p_{E}$ and hence they are equal. Thus, for any $x \in W$, we have $\|\psi(x)\|=$ $\|\psi \circ \phi(x)\|=\|F \circ E(x)\|=\|E(x)\|=\|\phi(x)\|$ and so $\phi$ is a minimal $V$-seminorm on $W$.

Hence, $\phi(W)=E(W)$ is a copy of $I_{W}(V)$. Now, in Theorem 2.11, it was shown that if $F$ is any map that extends $\phi$ and has minimal seminorm on $B(\mathcal{H})$ among all maps that extend $\phi$, then $F$ is a projection onto a copy of the injective envelope of $\phi(W)$. But, by the choice of $E$, it is minimal among all maps that extend $\phi=\left.E\right|_{W}$. Hence, $E(B(\mathcal{H}))$ is a copy of the injective envelope of $\phi(W)$ and hence is a copy of $I^{W}(V)$.

Remark 2.18. In Proposition 2.17, we are not asserting that such a minimal $p_{E}$ exists, only that when it does it has the asserted properties. However, if $V \subseteq W \subseteq$ $B(\mathcal{H})$ and $W$ is weak*-closed, then a $V$-map $E: B(\mathcal{H}) \rightarrow B(\mathcal{H})$ such that $p_{E}$ is minimal among all $V$-seminorms on $B(\mathcal{H})$ with $E(W) \subseteq W$ always exists. This can be seen by invoking Zorn's lemma. In this case any chain $\left\{E_{\lambda}\right\}$ will have a lower bound as can be seen by taking a weak*-limit point of the chain and noting that the limiting map $E$ will still satisfy $E(W) \subseteq W$.

Problem 2.19. Let $V \subseteq W \subseteq B(\mathcal{H})$ with $W$ admissible. Clearly, $I(V) \subseteq I^{W}(V)$. Are they always equal? Is it possible to give necessary and sufficient conditions that guarantee equality?

The following shows why we believe that the above problem is important.

Definition 2.20. A $C^{*}$-algebra $B$ is said to be QWEP (for quotient of WEP) if there is a $C^{*}$-algebra $A$ with WEP and a *-homomorphism from $A$ onto $B$.

Problem 2.21. Does a $C^{*}$-algebra $B \subseteq B(\mathcal{H})$ have QWEP if and only if $I^{B^{\prime \prime}}(B)=$ $I(B) ?$

In the next section we shall relate these quantities to questions about weak expectations.

\section{WEAK EXPECTATIONS AND MINIMAL PROJECTIONS}

We now turn our attention to some applications of the ideas of the previous section to the existence of weak expectations.

Definition 3.1. Given $V \subseteq B(\mathcal{H})$, an operator space, we shall let $\mathcal{E}(V)=\mathcal{E}_{B(\mathcal{H})}(V)$ denote the set of minimal $V$-projections on $B(\mathcal{H})$. Given $E \in \mathcal{E}(V)$, we call $\mathcal{R}(E)$ a copy of $I(V)$ and we denote the set of all operator spaces contained in $B(\mathcal{H})$ that are copies of $I(V)$ by $\mathcal{C I}(V)$.

Note that, in general, for each $\mathcal{S} \in \mathcal{C I}(V)$, there could be many projections, $E \in \mathcal{E}(V)$ with $S=\mathcal{R}(E)$. We will often use the following observation of Hamana [6], that if $E_{0}, E_{1} \in \mathcal{E}(V)$, then $E_{0} \circ E_{1} \in \mathcal{E}(V)$ since it must also define a minimal $V$-seminorm. Hence, $\mathcal{R}\left(E_{0} \circ E_{1}\right)=\mathcal{R}\left(E_{0}\right)$ and $E_{0}: \mathcal{R}\left(E_{1}\right) \rightarrow \mathcal{R}\left(E_{0}\right)$ is a complete isomorphism.

Given a concrete operator space $V \subseteq B(\mathcal{H})$, we shall let $V^{\dagger \dagger}$ denote the weak*closure of $V$ in $B(\mathcal{H})$, so that in the case that $V=\mathcal{A}$ is a $C^{*}$-subalgebra, we have that $\mathcal{A}^{\dagger \dagger}=\mathcal{A}^{\prime \prime}$. 
Theorem 3.2. Let $V \subseteq B(\mathcal{H})$ be an operator space. Then the following are equivalent:

(i) $V$ has a weak expectation.

(ii) $I_{V^{\dagger \dagger}}(V)=I(V)$; i.e., these spaces are completely isometrically isomorphic via a map that fixes $V$.

(iii) $I_{V^{\dagger \dagger}}(V)$ is injective, in the usual sense.

Proof. Assuming (ii), since $I(V)$ is injective we have (iii).

Assuming (iii), we have a projection onto $I_{V^{\dagger \dagger}}(V) \subseteq V^{\dagger \dagger}$, and so $V$ has a weak expectation. Thus, (iii) implies (i).

Finally, assuming (i), we have $E \in \mathcal{E}(V)$ with $\mathcal{R}(E) \subseteq V^{\dagger \dagger}$. Let $\phi: V^{\dagger \dagger} \rightarrow V^{\dagger \dagger}$ be any minimal $V$-projection, relative to $V^{\dagger \dagger}$ so that $\phi\left(V^{\dagger \dagger}\right)$ is a copy of $I_{V^{\dagger \dagger}}(V)$. Since the seminorm on $B(\mathcal{H})$ generated by $E$ is minimal among all $V$-seminorms, it is equal to the seminorm on $B(\mathcal{H})$ generated by $\phi \circ E$, and hence, $\phi \circ E \in \mathcal{E}(V)$. Also, $\phi$ : $E(B(\mathcal{H})) \rightarrow(\phi \circ E)(B(\mathcal{H}))$ is a complete isometry, since the two seminorms agree. By Theorem 2.9, $p_{\phi}$ is a minimal $V$-seminorm on $V^{\dagger \dagger}$. Hence, by Proposition 2.14, we have $(\phi \circ E)\left(V^{\dagger \dagger}\right)=\phi\left(V^{\dagger \dagger}\right)$, and so $(\phi \circ E)(B(\mathcal{H}))=(\phi \circ E)\left(V^{\dagger \dagger}\right)=\phi\left(V^{\dagger \dagger}\right)$. Thus, $\phi$ is a complete isometry from a copy of $I(V)$, namely $\mathcal{R}(E)$, onto a copy of $I_{V^{\dagger \dagger}}(V)$.

In the $C^{*}$-algebra case we can say a bit more.

Proposition 3.3. Let $\mathcal{A} \subseteq B(\mathcal{H})$ be a unital $C^{*}$-subalgebra. If $\mathcal{A}$ has a weak expectation, then $E\left(\mathcal{A}^{\prime \prime}\right)=\mathcal{R}(E)$ for every $E \in \mathcal{E}(\mathcal{A})$.

Proof. If $\mathcal{A}$ has a weak expectation, then there exists a copy of $I(\mathcal{A})$ inside $\mathcal{A}^{\prime \prime}$. Consequently, there exists $E_{0} \in \mathcal{E}(\mathcal{A})$ such that $\mathcal{R}\left(E_{0}\right) \subseteq \mathcal{A}^{\prime \prime}$. Since $E_{0}$ is a projection, $\mathcal{R}\left(E_{0}\right)=E_{0}\left(\mathcal{A}^{\prime \prime}\right)$. Now given any $E \in \mathcal{E}(\mathcal{A})$, we have that $\mathcal{R}(E)=\mathcal{R}\left(E \circ E_{0}\right)=$ $E\left(\mathcal{R}\left(E_{0}\right)\right)=E\left(E_{0}\left(\mathcal{A}^{\prime \prime}\right)\right) \subseteq E\left(\mathcal{A}^{\prime \prime}\right)$ and so, $E\left(\mathcal{A}^{\prime \prime}\right)=\mathcal{R}(E)$.

Problem 3.4. Let $\mathcal{A} \subseteq B(\mathcal{H})$ be a unital $C^{*}$-subalgebra. Does there exist $E \in$ $\mathcal{E}(\mathcal{A})$, such that $E\left(\mathcal{A}^{\prime \prime}\right)=\mathcal{R}(E) \cap \mathcal{A}^{\prime \prime}$ is a copy of $I_{\mathcal{A}^{\prime \prime}}(\mathcal{A})$ ?

If the answer to the above problem is affirmative, then the converse of the above proposition holds.

Let $\mathcal{K}(\mathcal{H})$ denote the ideal of compact operators on $\mathcal{H}$. We now turn our attention to the relationship between compact operators and weak expectations. Hamana 6 proves that if $\mathcal{A} \subseteq B(\mathcal{H})$ is a unital $C^{*}$-subalgebra and $\mathcal{K}(\mathcal{H}) \subset \mathcal{A}$, then $I(\mathcal{A})=$ $B(\mathcal{H})$. The following is a slight generalization and in the $C^{*}$-algebra case yields a different proof. This proof also serves to introduce some of the ideas of the next section.

Proposition 3.5. Let $\mathcal{K}(\mathcal{H}) \subseteq V \subseteq B(\mathcal{H})$ be an operator space. Then the identity map on $B(\mathcal{H})$ is the only $V$-map and, consequently, $I(V)=B(\mathcal{H})$.

Proof. Let $\phi$ be a $V$-map. We first show that $\phi(I)=I$, so that $\phi$ is a unital complete contraction and hence completely positive. Given operators, $A, B \in B(\mathcal{H})$, we use $(A, B)$ to denote the operator from $\mathcal{H} \oplus \mathcal{H}$ to $\mathcal{H}$ defined by $(A, B)\left(\begin{array}{l}h_{1} \\ h_{2}\end{array}\right)=A h_{1}+B h_{2}$.

To see this claim, note that for any finite rank projection $P$, since $\phi$ is completely contractive and $\phi(P)=P$, we have that $\|(P, \phi(I)-P)\|=\|(\phi(P), \phi(I-P))\| \leq$ $\|(P, I-P)\|=1$. Hence, $P P^{*}+(\phi(I)-P)(\phi(I)-P)^{*} \leq I$. Subtracting $P$ from both sides and then multiplying both sides by $P$ yields that $0 \leq P(\phi(I)-P)(\phi(I)-P) P \leq$ 
$P(I-P) P=0$. Hence, $0=P(\phi(I)-P)=P(\phi(I)-I)$. Since this holds for every finite rank projection, $\phi(I)=I$.

Every positive operator $R \in B(\mathcal{H})$ is the strong limit of an increasing net (sequence in the separable case) of finite rank positive operators, $\left\{F_{\alpha}\right\}$. Thus, we have that $F_{\alpha}=\phi\left(F_{\alpha}\right) \leq \phi(R)$. Taking limits, we have that $R \leq \phi(R)$. Choosing a scalar $r$ such that $0 \leq r I-R$ we have that $r I-R \leq \phi(r I-R)=r I-\phi(R)$ and hence $R=\phi(R)$. Since every operator is a sum of positive operators the result follows.

In the case of $C^{*}$-subalgebras, we can say something about the opposite extreme, $\mathcal{K}(\mathcal{H}) \cap \mathcal{A}=0$.

Proposition 3.6. Let $\mathcal{A} \subseteq B(\mathcal{H})$ be a unital $C^{*}$-subalgebra and assume that $\mathcal{K}(\mathcal{H}) \cap$ $\mathcal{A}=0$. Then for every copy $\mathcal{S}$ of $I(\mathcal{A})$, there exists a minimal $\mathcal{A}$-projection $E$ onto $\mathcal{S}$ with $E(\mathcal{K}(\mathcal{H}))=0$ and hence, $\mathcal{S} \cap \mathcal{K}(\mathcal{H})=(0)$. However, there can exist minimal $\mathcal{A}$-projections with $E(\mathcal{K}(\mathcal{H})) \neq 0$.

Proof. Consider the projection map $\pi$ of $B(\mathcal{H})$ onto the Calkin algebra, $\mathcal{Q}(\mathcal{H})$. Since this map is a ${ }^{*}$-isomorphism on $\mathcal{A}$, by rigidity, it must be a complete isometry on every copy of $I(\mathcal{A})$. Thus, by composition with $\pi$ one is able to obtain an $\mathcal{A}$ projection that vanishes on the compacts.

For an example of a minimal $\mathcal{A}$-projection onto a copy of $I(\mathcal{A})$ that does not vanish on the compacts, consider the case when $\mathcal{A}$ consists of the scalar multiples of the identity operator. Fix a unit vector $h \in \mathcal{H}$ and set $E(T)=\langle T h, h\rangle I$.

Problem 3.7. Does it also follow, in this case, that every copy of $I_{\mathcal{A}^{\prime \prime}}(\mathcal{A})$ and $I^{\mathcal{A}^{\prime \prime}}(\mathcal{A})$ intersects the compacts trivially?

In the case that $\mathcal{A}$ is irreducible, the above result can be improved.

Proposition 3.8. Let $\mathcal{A} \subseteq B(\mathcal{H})$ be a unital, irreducible $C^{*}$-subalgebra and assume that $\mathcal{K}(\mathcal{H}) \cap \mathcal{A}=0$. Then $\phi(\mathcal{K}(\mathcal{H})) \subseteq \mathcal{K}(\mathcal{H})$ for every $\mathcal{A}$-map $\phi$.

Proof. Let $P$ and $Q$ be fixed finite rank projections with $P Q=0$. By Kadison's transitivity theorem [9, Theorem 5.4.5], there exists a unitary $U \in \mathcal{A}$ such that $U=P-Q$ on the range of $P+Q$. Since $\|U\|=\|P-Q\|=1$, the range of $P+Q$ reduces $U$.

Let $H=\operatorname{Re}(U)$ and write $H=H^{+}-H^{-}$. Then $H^{+} \in \mathcal{A}, H^{+} \geq P$ and on the range of $P+Q, H^{+}=P$. Constructing one such element of $\mathcal{A}$ for each $Q$ and letting $Q$ tend strongly to $I-P$, we obtain a net of elements $H_{Q} \in \mathcal{A}$, such that $H_{Q} \geq P$ and converges strongly to $P$. Since $H_{Q}=\phi\left(H_{Q}\right) \geq \phi(P)$, we find that $P \geq \phi(P) \geq 0$.

Thus $\phi$ is rank reducing, and the result follows.

Proposition 3.9. Let $\mathcal{A} \subseteq B(\mathcal{H})$ be a unital, irreducible $C^{*}$-subalgebra and assume that $\mathcal{K}(\mathcal{H}) \cap \mathcal{A}=0$. If $E$ is a minimal $\mathcal{A}$-projection, then $E(\mathcal{K}(\mathcal{H}))=0$.

Proof. Let $\mathcal{S}$ be the range of $E$ and let $\gamma: \mathcal{Q}(\mathcal{H}) \rightarrow \mathcal{S}$ be a completely positive map with $\gamma(\pi(a))=a$ for every $a \in \mathcal{A}$. Since $p_{E}$ is a minimal $\mathcal{A}$-seminorm we have that $\gamma \circ \pi \circ E$ defines the same seminorm.

However, by the above result, this latter seminorm vanishes on the compact operators and hence $E$ must also vanish on the compact operators. 
For the next result, we need to recall the canonical decomposition of a completely bounded map, $\phi: B(\mathcal{H}) \rightarrow B(\mathcal{H})$ into a singular and absolutely continuous part, $\phi=\phi_{s}+\phi_{a c}$. This decomposition is achieved by considering the generalized Stinespring representation, $\phi(x)=V^{*} \pi(x) W$, where $\pi: B(\mathcal{H}) \rightarrow B\left(\mathcal{H}_{0}\right)$ is a *homomorphism and $V, W: \mathcal{H} \rightarrow \mathcal{H}_{0}$ are bounded linear maps and decomposing the *-homomorphism into its singular and absolutely continuous parts, $\pi=\pi_{s} \oplus \pi_{a c}$. Recall that this latter decomposition is obtained by setting $\mathcal{H}_{a c}=\pi(\mathcal{K}(\mathcal{H})) \mathcal{H}_{0}$ and $\mathcal{H}_{s}=\mathcal{H}_{0} \ominus \mathcal{H}_{a c}$. When $\phi$ is completely positive, it is easy to see that $\phi_{s}$ and $\phi_{a c}$ are also completely positive. We will also use the fact that $\phi_{a c}$ is weak*-continuous.

We also remind the reader that a selfadjoint algebra of operators $\mathcal{B}$ on a Hilbert space, $\mathcal{H}$, is said to act non-degenerately if the closed linear span $\mathcal{B H}$ is dense in $\mathcal{H}$.

Proposition 3.10. Let $\mathcal{A} \subseteq B(\mathcal{H})$ be a unital $C^{*}$-subalgebra and let $\phi$ be an $\mathcal{A}$ map. If $\mathcal{A} \cap \mathcal{K}(\mathcal{H})$ acts non-degenerately, then $\phi=\phi_{a c}$ and, consequently, $\phi$ is an $\mathcal{A}^{\prime \prime}$-map.

Proof. Since $\mathcal{A} \cap \mathcal{K}(\mathcal{H})$ is non-degenerate, we can find an increasing net of compact operators, $K_{i} \in \mathcal{A}$ which tend strongly to the identity. Since, $\phi_{s}\left(K_{i}\right)=0, K_{i}=$ $\phi_{a c}\left(K_{i}\right) \leq \phi_{a c}(I)$. But since these operators tend to the identity, $\phi_{a c}(I)=I$, and hence $\phi_{s}(I)=0$, which implies $\phi_{s}=0$, because it is completely positive.

Finally, since $\phi=\phi_{a c}$ is absolutely continuous and fixes $\mathcal{A}$, it must also fix $\mathcal{A}^{\prime \prime}$.

Lemma 3.11. Let $\mathcal{A} \subseteq B(\mathcal{H})$ be a unital $C^{*}$-subalgebra. If $\mathcal{A} \cap \mathcal{K}(\mathcal{H})$ acts nondegenerately, then $\mathcal{A}^{\prime \prime}$ is injective.

Proof. Let $\mathcal{B}=\mathcal{A} \cap \mathcal{K}(\mathcal{H})$. By [1, Theorem 1.4.5] and its proof, $\mathcal{B}$ is unitarily equivalent to the direct sum of elementary $C^{*}$-algebras where each algebra appears with certain multiplicities. Thus, after this unitary equivalence we have that $\mathcal{H}=\sum_{i} n_{i} \mathcal{H}_{i}$, where $n_{i}$ indicates the multiplicity with which $\mathcal{H}_{i}$ occurs and $\mathcal{B}=\left\{\sum_{i} \oplus n_{i} K_{i}: K_{i} \in \mathcal{K}\left(\mathcal{H}_{i}\right)\right\}$.

Since $\mathcal{B}=\mathcal{A} \cap \mathcal{K}(\mathcal{H})$, we have that after the unitary equivalence, each $A \in \mathcal{A}$ is necessarily of the form $A=\sum_{i} \oplus n_{i} A_{i}$, with $A_{i} \in B\left(\mathcal{H}_{i}\right)$. Hence, $\mathcal{B}^{\prime \prime} \subseteq \mathcal{A}^{\prime \prime} \subseteq$ $\left\{\sum_{i} n_{i} B_{i}: B_{i} \in B\left(\mathcal{H}_{i}\right), \sup _{i}\left\|B_{i}\right\|<\infty\right\}=\mathcal{B}^{\prime \prime}$, and it follows that

$$
\mathcal{A}^{\prime \prime}=\mathcal{B}^{\prime \prime}=\left\{\sum_{i} \oplus n_{i} B_{i}: B_{i} \in B\left(\mathcal{H}_{i}\right), \sup _{i}\left\|B_{i}\right\|<\infty\right\},
$$

which is clearly injective.

Theorem 3.12. Let $\mathcal{A} \subseteq B(\mathcal{H})$ be a unital $C^{*}$-subalgebra. If $\mathcal{A} \cap \mathcal{K}(\mathcal{H})$ acts non-degenerately and $E \in \mathcal{E}(\mathcal{A})$, then $E$ is weak ${ }^{*}$-continuous and $\mathcal{R}(E)=\mathcal{A}^{\prime \prime}$. Consequently, $I(\mathcal{A})=\mathcal{A}^{\prime \prime}$ and $\mathcal{A}^{\prime \prime}$ is the unique copy of $I(\mathcal{A})$ contained in $B(\mathcal{H})$.

Proof. By Proposition 3.10, $E=E_{a c}$ so that $E$ is weak*-continuous and also $E$ fixes $\mathcal{A}^{\prime \prime}$ so that $\mathcal{A}^{\prime \prime} \subseteq \mathcal{R}(E)$. But since $\mathcal{A}^{\prime \prime}$ is injective, $\mathcal{R}(E) \subseteq \mathcal{A}^{\prime \prime}$ for any minimal $\mathcal{A}$-projection and the result follows.

Earlier, we saw that if we fix $E \in \mathcal{E}(\mathcal{A})$, then the set $\mathcal{J}_{E}$ is a convex left ideal in the semigroup of all $\mathcal{A}$-maps. Note that in this case $\mathcal{J}_{E}$ is also closed in the pointweak*-topology. We also have that $\mathcal{J}_{E}$ is left invariant under the action of the smaller convex semigroup $\Gamma$ of normal $\mathcal{A}$-maps. Consequently, there exist minimal $\Gamma$-invariant subsets of $\mathcal{E}(\mathcal{A})$. 
Proposition 3.13. Let $\mathcal{A} \subseteq B(\mathcal{H})$ be a unital $C^{*}$-subalgebra. Then $\mathcal{A}$ has a weak expectation if and only if there exists a minimal $\Gamma$-invariant subset of $\mathcal{E}(\mathcal{A})$ that is a singleton.

Proof. Let $\{E\}$ be a $\Gamma$-invariant subset that is a singleton and let $U \in \mathcal{A}^{\prime}$ be a unitary. Then $U^{*} E(x) U=E(x)$ for all $x$ and it follows that the range of $E$ is contained in $\mathcal{A}^{\prime \prime}$.

Conversely, by a theorem of Haagerup [5] (for a published proof, see Smith [12]) every element of $\Gamma$ has the form $\phi(x)=\sum b_{i}^{*} x b_{i}$ for some sequence of elements in $\mathcal{A}^{\prime}$ satisfying $\sum b_{i}^{*} b_{i}=1$. If $\mathcal{A}$ has a weak expectation, then there is a minimal $\mathcal{A}$-projection $E$, whose range is contained in $\mathcal{A}^{\prime \prime}$, and $E$ is easily seen to be fixed by $\Gamma$.

If $\mathcal{A}$ has a weak expectation, does every minimal $\Gamma$-invariant subset have to be a singleton?

\section{THE FIXED SPACE}

In this section we study the set of elements that are fixed by all $V$-maps. We first show that this is a type of reflexive cover of $V$ and then in the case of a $C^{*}$ subalgebra, we show that this set can be identified with a type of order completion.

Definition 4.1. Let $V \subseteq W$ be operator spaces. We set $\mathcal{F}_{W}(V)=\{T \in W$ : $\phi(T)=T$ for every $V$-map, $\phi: W \rightarrow W\}$. When $V \subseteq B(\mathcal{H})$ we shall write $\mathcal{F}(V) \equiv$ $\mathcal{F}_{B(\mathcal{H})}(V)$.

We shall generally be concerned with the case where $V=\mathcal{A}$ and $W=\mathcal{B}$ are $C^{*}$-algebras, but the case of a pair of operator spaces is equally interesting. The following is immediate.

Proposition 4.2. Let $V \subseteq W \subseteq \mathcal{B}(\mathcal{H})$. Then $\mathcal{F}(V) \cap W \subseteq \mathcal{F}_{W}(V)$.

In general we won't have equality. To see this note that if $\mathcal{A} \subseteq \mathcal{S} \subseteq B(\mathcal{H})$, where $\mathcal{S}$ is one of the copies of $I(\mathcal{A})$, then $\mathcal{F}_{\mathcal{S}}(\mathcal{A})=\mathcal{S}$, since every completely positive map from $\mathcal{S}$ to $\mathcal{S}$ that fixes $\mathcal{A}$ necessarily fixes all of $\mathcal{S}$, by the rigidity property of injective envelopes. But there can be other copies of the injective envelope embedded in $B(\mathcal{H})$ and, by taking a projection onto one of these other copies, we obtain a completely positive map on $B(\mathcal{H})$ that fixes $\mathcal{A}$ but doesn't fix $\mathcal{S}$.

The following result shows that $\mathcal{F}(V)$ is a sort of reflexive cover of $V$.

Proposition 4.3. Let $V \subseteq B(\mathcal{H})$ be an operator space. Then $\mathcal{F}(V)=\{T \in$ $B(\mathcal{H}): E(T)=T$ for every $E \in \mathcal{E}(V)\}=\bigcap \mathcal{S}$, where the intersection is taken over all $\mathcal{S} \in \mathcal{C} \mathcal{I}(V)$, i.e., over all copies of $I(V)$. Consequently, $I(V)=I(\mathcal{F}(V))$.

Proof. The equality of the last two sets is obvious, as is the fact that the first set is contained in the second set. Now if $E(T)=T$ for every $E \in \mathcal{E}(V)$ and $\phi$ is any $V$-map, then $\phi(T)=\phi(E(T))=T$, since $\phi \circ E \in \mathcal{E}(V)$.

In the case of a $C^{*}$-subalgebra we can say a bit more.

Proposition 4.4. Let $\mathcal{A} \subseteq B(\mathcal{H})$ be a unital $C^{*}$-subalgebra. Then $\mathcal{F}(\mathcal{A}) \subseteq$ $\mathcal{F}_{\mathcal{A}^{\prime \prime}}(\mathcal{A}) \subseteq \mathcal{A}^{\prime \prime}$. Moreover, $I_{\mathcal{A}^{\prime \prime}}(\mathcal{A})=I_{\mathcal{A}^{\prime \prime}}(\mathcal{F}(\mathcal{A}))$. 
Proof. Let $P \in \mathcal{A}^{\prime}$ be a projection and define an $\mathcal{A}$-map by $\phi(X)=P X P+(I-$ $P) X(I-P)$. If $T \in \mathcal{F}(\mathcal{A})$, then $\phi(T)=T$ and hence $T P=P T$. Since $T$ commutes with every projection in $\mathcal{A}^{\prime}$, we have that $T \in \mathcal{A}^{\prime \prime}$. Thus, $\mathcal{F}(\mathcal{A}) \subseteq \mathcal{A}^{\prime \prime}$. Applying Proposition 4.2, we have that $\mathcal{F}(\mathcal{A})=\mathcal{F}(\mathcal{A}) \cap \mathcal{A}^{\prime \prime} \subseteq \mathcal{F}_{\mathcal{A}^{\prime \prime}}(\mathcal{A})$.

The last statement follows since any unital completely positive map that fixes $\mathcal{A}$ fixes $\mathcal{F}(\mathcal{A})$.

Problem 4.5. Is $\mathcal{F}(V) \subseteq V^{\dagger \dagger}$ for every operator space?

Remark 4.6. Note that by the above results, if $\mathcal{A}=\mathcal{A}^{\prime \prime} \subseteq B(\mathcal{H})$ is a non-injective von Neumann subalgebra, then $\mathcal{A}=\mathcal{F}(\mathcal{A})=\bigcap \mathcal{S}$ and so there are certainly multiple copies of $I(\mathcal{A})$. Later we will see an example of a non-injective $C^{*}$-algebra for which there is a unique copy of $I(\mathcal{A})$ and $I(\mathcal{A}) \subsetneq \mathcal{A}^{\prime \prime}$.

Proposition 4.7. Let $\mathcal{A} \subseteq B(\mathcal{H})$ be a unital $C^{*}$-subalgebra. If $\mathcal{A} \cap \mathcal{K}(\mathcal{H})$ acts non-degenerately, then $\mathcal{F}(\mathcal{A})=\mathcal{A}^{\prime \prime}$.

Proof. By Theorem $3.12, \mathcal{A}^{\prime \prime} \subseteq \mathcal{F}(\mathcal{A})$.

Proposition 4.8. Let $\mathcal{A} \subseteq B(\mathcal{H})$ be a unital $C^{*}$-subalgebra. If $\mathcal{A} \cap \mathcal{K}(\mathcal{H})=(0)$, then $\mathcal{F}(\mathcal{A}) \cap \mathcal{K}(\mathcal{H})=(0)$.

Proof. Apply Proposition 3.6.

The next result shows that many of the various notions of multipliers that can be associated with $\mathcal{A}$ are in $\mathcal{F}(\mathcal{A})$.

Definition 4.9. Let $\mathcal{A} \subseteq B(\mathcal{H})$ be a unital $C^{*}$-subalgebra. An operator $T \in B(\mathcal{H})$ is called a local left multiplier of $\mathcal{A}$, provided that there exists a two-sided ideal $\mathcal{J} \triangleleft \mathcal{A}$ of $\mathcal{A}$ that acts non-degenerately on $\mathcal{H}$, such that $T \cdot \mathcal{J} \subseteq \mathcal{A}$. Similarly, $T$ is called a local right multiplier of $\mathcal{A}$ if there exists such an ideal with $\mathcal{J} \cdot T \subseteq \mathcal{A}$, and a local quasi-multiplier of $\mathcal{A}$, provided that there is such an ideal with $\mathcal{J} \cdot T \cdot \mathcal{J} \subseteq \mathcal{A}$. These sets of operators are denoted by $\mathcal{L M}_{\text {loc }}(\mathcal{A}), \mathcal{R M}_{\text {loc }}(\mathcal{A})$ and $\mathcal{Q} \mathcal{M}_{\text {loc }}(\mathcal{A})$, respectively.

It is fairly easy to check that each of these sets of operators is a vector space and that $\mathcal{L M}_{\text {loc }}(\mathcal{A}) \cup \mathcal{R} \mathcal{M}_{\text {loc }}(\mathcal{A}) \subseteq \mathcal{Q M}_{\text {loc }}(\mathcal{A})$. Moreover, $T \in \mathcal{Q M}_{\text {loc }}(\mathcal{A})$ if and only if $\operatorname{Re}(T), \operatorname{Im}(T) \in \mathcal{Q} \mathcal{M}_{\text {loc }}(\mathcal{A})$.

Proposition 4.10. Let $\mathcal{A} \subseteq B(\mathcal{H})$ be a unital $C^{*}$-subalgebra. Then $\mathcal{Q M}_{\text {loc }}(\mathcal{A}) \subseteq$ $\mathcal{F}(\mathcal{A})$.

Proof. Let $T \in \mathcal{Q M}_{\text {loc }}(\mathcal{A})$ and let $\mathcal{J} \triangleleft \mathcal{A}$ be a two-sided ideal that acts nondegenerately on $\mathcal{H}$, with $\mathcal{J} \cdot T \cdot \mathcal{J} \subseteq \mathcal{A}$. We wish to show that $T \in \mathcal{F}(\mathcal{A})$.

Since $\mathcal{J}$ acts non-degenerately, there is an increasing, positive, approximate identity $\left\{e_{\alpha}\right\}$ for $\mathcal{J}$ that tends strongly to $I$. Let $\phi$ be any $\mathcal{A}$-map, so that $\phi$ is a unital completely positive map that fixes $\mathcal{A}$.

By Choi's theory of multiplicative domains $4, \phi$ is an $\mathcal{A}$-bimodule map. Hence, for each $\alpha$ and $\beta,\left(e_{\alpha} T-e_{\alpha} \phi(T)\right) e_{\beta}=0$. Using that $e_{\beta}$ tends strongly to $I$ yields that $e_{\alpha} T=e_{\alpha} \phi(T)$, for each $\alpha$. Now using that $e_{\alpha}$ tends strongly to $I$ yields $T=\phi(T)$. Thus, $T \in \mathcal{F}(\mathcal{A})$ as was to be shown.

We now show that $\mathcal{F}(\mathcal{A})$ is in a certain sense an order completion of $\mathcal{A}$. 
Definition 4.11. Let $T=T^{*} \in B(\mathcal{H})$. Then we set $(-\infty, T]_{\mathcal{A}}=\left\{A=A^{*} \in\right.$ $\mathcal{A}: A \leq T\}$ and set $[T,+\infty)_{\mathcal{A}}=\left\{A=A^{*} \in \mathcal{A}: T \leq A\right\}$. For $R=R^{*}$, we write $(-\infty, T]_{\mathcal{A}} \leq R$ provided that $A \leq R$ for every $A \in(-\infty, T]_{\mathcal{A}}$ and define $R \leq[T,+\infty)_{\mathcal{A}}$, similarly. We say that $T$ is order determined by $\mathcal{A}$ if $(-\infty, T]_{\mathcal{A}} \leq$ $R \leq[T,+\infty)_{\mathcal{A}}$ implies that $T=R$.

We say that $T$ is matricially order determined by $\mathcal{A}$ provided that $(-\infty, T \otimes$ $H]_{M_{n}(\mathcal{A})} \leq R \otimes H \leq[T \otimes H,+\infty)_{M_{n}(\mathcal{A})}$ for every $n$ and every $H=H^{*} \in M_{n}$ implies that $R=T$.

Note that the set of order determined elements is a subset of the matricially order determined elements.

Proposition 4.12. Let $\mathcal{A} \subseteq B(\mathcal{H})$ be a unital $C^{*}$-subalgebra and let $T=T^{*} \in$ $B(\mathcal{H})$. Then $T \in \mathcal{F}(\mathcal{A})$ if and only if $T$ is matricially order determined by $\mathcal{A}$.

Proof. Let $\phi$ be any $\mathcal{A}$-map. It is easily seen that $(-\infty, T \otimes H]_{M_{n}(\mathcal{A})} \leq \phi(T) \otimes H \leq$ $[T \otimes H,+\infty)_{M_{n}(\mathcal{A})}$ for every $n$ and every $H=H^{*} \in M_{n}$. Hence, if $T$ is matricially order determined, then $\phi(T)=T$ and so $T \in \mathcal{F}(\mathcal{A})$.

Conversely, assume that $T \in \mathcal{F}(\mathcal{A})$ and assume that $R=R^{*}$ satisfies the inequalities in the definition. These inequalities imply that there is a well-defined completely positive map, $\phi$, satisfying $\phi(A+\lambda T)=A+\lambda R$ from the operator system spanned by $\mathcal{A}$ and $T$ onto the operator system spanned by $\mathcal{A}$ and $R$. This completely positive map can then be extended to a completely positive map on all of $B(\mathcal{H})$, and hence $R=\phi(T)=T$.

Maitland Wright 13, 14, 15 and Hamana 8 studied several different monotone completions of a $C^{*}$-algebra. In spite of the above characterization of $\mathcal{F}(\mathcal{A})$, we have been unable to develop any relationship between $\mathcal{F}(\mathcal{A})$ and those other completions.

Remark 4.13. The set $\mathcal{F}(\mathcal{A})$ is not generally a $C^{*}$-subalgebra of $B(\mathcal{H})$ as we will show below.

We now wish to recall another construction of Hamana's [8]. Given a concrete operator space, $V \subset B(\mathcal{H})$, we have a new operator space $\widehat{V} \subseteq B\left(\mathcal{H} \otimes \ell^{2}\right)$ defined as follows. Every $T \in B\left(\mathcal{H} \otimes \ell^{2}\right)$ has the form $T=\left(T_{i, j}\right)$, with $T_{i, j} \in B(\mathcal{H})$. We set $\widehat{V}=\left\{\left(T_{i, j}\right) \in B\left(\mathcal{H} \otimes \ell^{2}\right): T_{i, j} \in V\right\}$. This is Hamana's Fubini product of $V$ with $B\left(\ell^{2}\right)$.

If $\phi: B(\mathcal{H}) \rightarrow B(\mathcal{H})$ is completely bounded, then $\widehat{\phi}: B\left(\mathcal{H} \otimes \ell^{2}\right) \rightarrow B\left(\mathcal{H} \otimes \ell^{2}\right)$, defined by $\widehat{\phi}\left(\left(T_{i, j}\right)\right)=\left(\phi\left(T_{i, j}\right)\right)$ is also completely bounded with $\|\phi\|_{c b}=\|\widehat{\phi}\|_{c b}$. Hence, if $V$ is injective and $\phi: B(\mathcal{H}) \rightarrow V$ is a completely contractive projection onto $V$, then $\widehat{\phi}: B\left(\mathcal{H} \otimes \ell^{2}\right) \rightarrow \widehat{V}$ is a completely contractive projection onto $\widehat{V}$ and so $\widehat{V}$ is also injective.

We now wish to define another operator space associated with $V$. First, let $\ell^{\infty}(V)$ denote the subset of $\widehat{V}$ consisting of diagonal matrices with entries from $V$ and let $V \otimes \mathcal{K}\left(\ell^{2}\right) \subseteq \widehat{V}$ denote the tensor product of $V$ and the compact operators on $\ell^{2}, \mathcal{K}\left(\ell^{2}\right)$. Finally, we let $\widetilde{V}=\ell^{\infty}(V)+V \otimes \mathcal{K}\left(\ell^{2}\right)$.

Proposition 4.14. Let $\mathcal{A} \subseteq B(\mathcal{H})$ be a unital $C^{*}$-subalgebra. Then $\widetilde{\mathcal{A}} \subseteq B\left(\mathcal{H} \otimes \ell^{2}\right)$ is a $C^{*}$-subalgebra. If $\Psi: B\left(\mathcal{H} \otimes \ell^{2}\right) \rightarrow B\left(\mathcal{H} \otimes \ell^{2}\right)$ is a unital, completely positive map that fixes $\widetilde{\mathcal{A}}$, then there exists $\phi: B(\mathcal{H}) \rightarrow B(\mathcal{H})$ that fixes $\mathcal{A}$ such that $\Psi=\widehat{\phi}$. 
Proof. Since $\Psi$ fixes the $C^{*}$-algebra, $\mathcal{C}=\ell^{\infty}(\mathbb{C} \cdot I)+\left(\mathbb{C} \cdot I \otimes \mathcal{K}\left(\ell^{2}\right)\right)$, we have that $\Psi$ must be a $\mathcal{C}$-bimodule map and, hence, must be of the form $\Psi=\widehat{\phi}$ for some map $\phi$. The result now follows easily.

Theorem 4.15. Let $\mathcal{A} \subseteq B(\mathcal{H})$ be a unital $C^{*}$-subalgebra. Then $\Psi \in \mathcal{E}(\widetilde{\mathcal{A}})$ if and only if $\Psi=\widehat{\phi}$ for some $\phi \in \mathcal{E}(\mathcal{A})$ and $\mathcal{F}(\widetilde{\mathcal{A}})=\widehat{\mathcal{F}(\mathcal{A})}$.

Proof. The fact that $\Psi=\widehat{\phi}$ for $\phi \in \mathcal{E}(\mathcal{A})$ follows from the above proposition. Note that in this case, $\mathcal{R}(\Psi)=\widehat{\mathcal{R}(\phi)}$, and the result follows from the characterization of $\mathcal{F}(\cdot)$ as the intersection of all ranges.

Corollary 4.16. Let $\mathcal{A} \subseteq B(\mathcal{H})$ be a unital injective $C^{*}$-subalgebra. Then $\widetilde{\mathcal{A}} \subseteq$ $B\left(\mathcal{H} \otimes \ell^{2}\right)$ is not injective, and $\mathcal{F}(\widetilde{\mathcal{A}})=I(\widetilde{\mathcal{A}})=\widehat{\mathcal{A}} \subseteq B\left(\mathcal{H} \otimes \ell^{2}\right)$ is the unique copy of its injective envelope.

We now wish to show that it is possible to find an abelian, injective $C^{*}$-subalgebra, $\mathcal{A} \subseteq B(\mathcal{H})$, such that $\widehat{\mathcal{A}} \subseteq B\left(\mathcal{H} \otimes \ell^{2}\right)$ is not a $C^{*}$-subalgebra. First, we will need some preliminary results which might be of independent interest.

Let $X$ be a compact, Hausdorff space. If we identify $I(C(X))=C(Y)$ for some compact, Hausdorff space $Y$, then the inclusion of $C(X)$ in $C(Y)$ is induced by a continuous, onto function, $p: Y \rightarrow X$. Assume that $\left\{x_{n}\right\}$ is a countable, dense subset of $X$ and choose $y_{n} \in Y$ such that $p\left(y_{n}\right)=x_{n}$. In the following sequence of results we assume that this situation holds.

Proposition 4.17. Define $\pi: C(Y) \rightarrow \ell^{\infty}$ by $\pi(f)=\left(f\left(y_{n}\right)\right)$. Then $\pi$ is a one-toone *-homomorphism and, consequently, $\left\{y_{n}\right\}$ is dense in $Y$.

Proof. Clearly, $\pi$ is a *-homomorphism. Define $\rho: C(X) \rightarrow \ell^{\infty}$ by $\rho(f)=\left(f\left(x_{n}\right)\right)$. Since $\left\{x_{n}\right\}$ is dense in $X, \rho$ is a one-to-one *homomorphism. Also, since $p\left(y_{n}\right)=$ $x_{n}, \pi$ is an extension of $\rho$ to $C(Y)$, i.e., $\rho=\pi \circ p^{*}$.

But since $C(Y)$ is an essential extension of $C(X)$, the fact that $\rho$ is isometric forces $\pi$ to be isometric and hence $\left\{y_{n}\right\}$ must be a dense subset of $Y$.

The above proof gives one of the easiest proofs of the following result.

Corollary 4.18. Let $X$ and $Y$ be compact, Hausdorff spaces, such that $C(Y)$ is $C^{*}$-isomorphic to $I(C(X))$. If $X$ is separable, then $Y$ is separable.

Now let $X=[0,1]$ and let $\left\{x_{n}\right\}$ be a dense subset as above of distinct points and for convenience we let $x_{1}=1$. Note that $\rho(C([0,1])) \cap c_{0}=(0)$, for if $\rho(f) \in c_{0}$, then given any $x \in[0,1]$ we could choose a subsequence $\left\{x_{n_{k}}\right\}$ with $\lim _{k} x_{n_{k}}=x$ and hence $f(x)=\lim _{k} f\left(x_{n_{k}}\right)=0$.

Thus, if we let $\tilde{\rho}: C([0,1]) \rightarrow \ell^{\infty} / c_{0}$ denote the composition of $\rho$ with the quotient map, then $\tilde{\rho}$ is still one-to-one and hence an isometry. Thus, again by the fact that $C(Y)$ is an essential extension of $C([0,1])$, we have that the composition of $\pi$ with the quotient map $\tilde{\pi}: C(Y) \rightarrow \ell^{\infty} / c_{0}$ is also an isometry and hence one-to-one.

Lemma 4.19. There exists an injective, $C^{*}$-subalgebra $\mathcal{B}$ of $\ell^{\infty}$ such that $\mathcal{B} \cap c_{0}=$ $(0), \mathcal{B}$ is weak ${ }^{*}$-dense in $\ell^{\infty}$ and for every $n$, there exists a strictly positive element, $b_{n} \in \mathcal{B}$ such that $\lim _{m} b_{n}^{m}=\delta_{n}$, pointwise, where $\delta_{n}$ is the function that is 1 at $n$ and 0 elsewhere. 
Proof. Let $\mathcal{B}=\pi(C(Y))$, where $C(Y)$ is the injective envelope of $C([0,1])$ as above. Then $\mathcal{B}$ is injective and as shown above, $\mathcal{B} \cap c_{0}=(0)$.

Next, for each point $x_{n} \in[0,1]$ choose a strictly positive continuous function $f_{n}$ such that $1=f_{n}\left(x_{n}\right)>f_{n}(x) \geq 1 / 2$ for any $x \neq x_{n}$ and let $b_{n}=\rho\left(f_{n}\right)$.

The existence of such $b_{n}$ shows that each $\delta_{n}$ is in the weak*-closure of $\mathcal{B}$ and, hence, $\mathcal{B}$ is weak ${ }^{*}$-dense.

The key to the next result is that products in $\widehat{\mathcal{B}}$ involve strong convergence of sums while injective $C^{*}$-subalgebras need not be closed in the strong operator topology.

Theorem 4.20. Let $\mathcal{B} \subseteq \ell^{\infty}$ be the $C^{*}$-subalgebra of Lemma 4.19 and assume that $\ell^{\infty} \subseteq B\left(\ell^{2}\right)$ is represented as the diagonal operators. Then $\mathcal{F}(\widetilde{\mathcal{B}})=I(\widetilde{\mathcal{B}})=\widehat{\mathcal{B}}$ is not a $C^{*}$-subalgebra of $B\left(\mathcal{H} \otimes \ell^{2}\right)$.

Proof. Since $\mathcal{B}$ is injective, $\mathcal{F}(\mathcal{B})=\mathcal{B}$, and hence, by Theorem $4.15, \mathcal{F}(\widetilde{\mathcal{B}})=\widehat{\mathcal{F}(\mathcal{B})}=$ $\widehat{\mathcal{B}}$. Thus, it remains to show that even though $\mathcal{B}$ is an injective $C^{*}$-subalgebra of $B\left(\ell^{2}\right), \widehat{\mathcal{B}}$ is not a $C^{*}$-subalgebra of $B\left(\ell^{2} \otimes \ell^{2}\right)$, although by the above results it is an injective operator system.

To this end, let $b_{1}$ be the element of $\mathcal{B}$ that satisfies $1=b_{1}(1)>b_{1}(n) \geq 1 / 2$, for $n \neq 1$. Set $P_{1}=\sqrt{b_{1}}$ and for $n>1$, set $P_{n}=\sqrt{b_{1}^{n-1}-b_{1}^{n}}$. Let $A=\left(A_{i, j}\right), B=$ $\left(B_{i, j}\right)$ be defined by $A_{1, j}=P_{j}, A_{i, j}=0, i \neq 1$ and $B_{1,1}=P_{1}, B_{i, 1}=-P_{i}, i \neq 1$, while $B_{i, j}=0, j \neq 1$. Then, since $\sum_{k \geq 1} P_{k}^{2} \leq 2 b_{1}$, we have that $A$ and $B$ define bounded operators and hence are in $\widehat{\mathcal{B}}$.

However, $A \cdot B=\left(C_{i, j}\right)$, where $C_{i, j}=0$, unless $i=j=1$ and $C_{1,1}=P_{1}^{2}-$ $\sum_{k \geq 2} P_{k}^{2}=\lim _{k \rightarrow \infty} b_{1}^{k}=\delta_{1}$ since all convergence is only in the strong operator topology. However, $\delta_{1} \notin \mathcal{B}$ by construction. Hence, $\widehat{\mathcal{B}}$ is not a $C^{*}$-subalgebra.

Note that for the above example, $\mathcal{F}(\widetilde{\mathcal{B}})=\widehat{\mathcal{B}}=I(\widetilde{\mathcal{B}})$. Thus, although $\mathcal{F}(\widetilde{\mathcal{B}})$ is not a $C^{*}$-subalgebra of $B\left(\mathcal{H} \otimes \ell^{2}\right)$, it is an injective operator system and it is a $C^{*}$-algebra in another product.

Problem 4.21. Is $\mathcal{F}(\mathcal{A})$ always completely order isomorphic to a $C^{*}$-algebra?

In particular, there is a natural way to identify $\mathcal{F}(\mathcal{A})$ completely order isomorphically with an operator subsystem of $I(\mathcal{A})$, and we conjecture that it is a $C^{*}$-subalgebra of $I(\mathcal{A})$ with this identification. We make this precise below.

Definition 4.22. Let $\mathcal{A}$ be a unital $C^{*}$-algebra and let $\pi: \mathcal{A} \rightarrow B(\mathcal{H})$ be a *-monomorphism. We let $\mathcal{E}(\pi)$ denote the set of all completely positive maps $\phi$ : $I(\mathcal{A}) \rightarrow B(\mathcal{H})$ that extend $\pi$ and we set $\mathcal{F}_{\pi}=\{x \in I(\mathcal{A}): \phi(x)=\psi(x)$ for all $\phi, \psi \in$ $\mathcal{E}(\pi)\}$.

Proposition 4.23. Let $\mathcal{A}$ be a unital $C^{*}$-algebra and let $\pi: \mathcal{A} \rightarrow B(\mathcal{H})$ be a ${ }^{*}$-monomorphism. If $\phi \in \mathcal{E}(\pi)$, then $\phi: \mathcal{F}_{\pi} \rightarrow \mathcal{F}(\pi(\mathcal{A}))$ is a complete order isomorphism.

Proof. Clearly, $E(T)=T$ for every $E \in \mathcal{E}(\pi(\mathcal{A}))$ if and only if $T=\phi(x)$ for a unique element of $\mathcal{F}_{\pi}$.

Problem 4.24. Is $\mathcal{F}_{\pi} \subseteq I(\mathcal{A})$, always a $C^{*}$-subalgebra? 
We close this section by examining the above construction in the abelian case. Recall that an abelian $C^{*}$-algebra is injective if and only if it is an $\mathrm{AW}^{*}$-algebra [6] and that, in an abelian $\mathrm{AW}^{*}$-algebra, every set of selfadjoint elements with an upper bound (respectively, lower bound) has a supremum (respectively, infimum). Also, every abelian $\mathrm{W}^{*}$-algebra is injective. Given any subset $S$ of a $C^{*}$-algebra, we let $S_{h}=\left\{x \in S: x=x^{*}\right\}$.

Given an abelian, injective $C^{*}$-algebra $\mathcal{C}$ and a $C^{*}$-subalgebra $\mathcal{A}$, we follow Hamana's notation 8 and given $x \in \mathcal{C}_{h}$ set

$$
(-\infty, x]_{\mathcal{A}}=\left\{a \in \mathcal{A}_{h}: a \leq x\right\}
$$

and

$$
[x,+\infty)_{\mathcal{A}}=\left\{a \in \mathcal{A}_{h}: x \leq a\right\} .
$$

Moreover, we shall set

$$
\ell_{\mathcal{A}}(x)=\sup (-\infty, x]_{\mathcal{A}}
$$

and

$$
u_{\mathcal{A}}(x)=\inf [x,+\infty)_{\mathcal{A}} .
$$

Theorem 4.25. Let $\mathcal{A} \subset \mathcal{B}(\mathcal{H})$ be a unital abelian $C^{*}$-subalgebra with $\mathcal{A}^{\prime}=\mathcal{A}^{\prime \prime}$. Then $\mathcal{F}(\mathcal{A})_{h}=\mathcal{F}_{\mathcal{A}^{\prime \prime}}(\mathcal{A})_{h}=\left\{x \in \mathcal{A}_{h}^{\prime \prime}: \ell_{\mathcal{A}}(x)=u_{\mathcal{A}}(x)\right\}$, where $\ell_{\mathcal{A}}(x)$ and $u_{\mathcal{A}}(x)$ are computed in $\mathcal{A}^{\prime \prime}$.

Proof. Let $E: B(\mathcal{H}) \rightarrow \mathcal{A}^{\prime \prime}$ be a completely positive projection. Since $E$ fixes $\mathcal{A}$, if $x \in \mathcal{F}(\mathcal{A})$, then $E(x)=x$ and so $x \in \mathcal{A}^{\prime \prime}$. Thus, $\mathcal{F}(\mathcal{A})=\mathcal{F}(\mathcal{A}) \cap \mathcal{A}^{\prime \prime} \subseteq \mathcal{F}_{\mathcal{A}^{\prime \prime}}(\mathcal{A})$.

Now, if $x \in \mathcal{A}_{h}^{\prime \prime}$ and $\ell_{\mathcal{A}}(x) \neq u_{\mathcal{A}}(x)$, then there exists $y \in \mathcal{A}_{h}^{\prime \prime}$ such that $\ell_{\mathcal{A}}(x) \leq$ $y \leq u_{\mathcal{A}}(x)$ with $y \neq x$.

It is easily checked that for any $a \in \mathcal{A}$ and $\lambda \in \mathbb{C}$, we have that $a+\lambda x \geq 0$ implies that $a+\lambda y \geq 0$. Hence, the map $\phi(a+\lambda x)=a+\lambda y$ is positive and, since we are in an abelian situation, completely positive. Thus, we may extend $\phi$ to a map $\psi: \mathcal{A}^{\prime \prime} \rightarrow \mathcal{A}^{\prime \prime}$. Since $\psi$ fixes $\mathcal{A}$ and $\psi(x)=y$, we have that $x \notin \mathcal{F}_{\mathcal{A}^{\prime \prime}}(\mathcal{A})$. Thus, we have shown that $\mathcal{F}(\mathcal{A})_{h} \subseteq \mathcal{F}_{\mathcal{A}^{\prime \prime}}(\mathcal{A})_{h} \subseteq\left\{x \in \mathcal{A}_{h}^{\prime \prime}: \ell_{\mathcal{A}}(x)=u_{\mathcal{A}}(x)\right\}$.

Now assume that $x \in \mathcal{A}_{h}^{\prime \prime}$ and $\ell_{\mathcal{A}}(x)=u_{\mathcal{A}}(x)$. If $\phi: B(\mathcal{H}) \rightarrow B(\mathcal{H})$ is any completely positive map such that $\phi(a)=a \forall a \in \mathcal{A}$, then $\phi\left(\mathcal{A}^{\prime}\right) \subseteq \mathcal{A}^{\prime}$, since for $y \in \mathcal{A}^{\prime}, a \phi(y)=\phi(a y)=\phi(y a)=\phi(y) a$. Thus, if $\phi(x)=y$, then $y \in \mathcal{A}^{\prime \prime}$ and hence, $\ell_{\mathcal{A}}(x) \leq y \leq u_{\mathcal{A}}(x)$ from which it follows that $y=x$. Hence, $\left\{x \in \mathcal{A}_{h}^{\prime \prime}: \ell_{\mathcal{A}}(x)=\right.$ $\left.u_{\mathcal{A}}(x)\right\} \subseteq \mathcal{F}(\mathcal{A})$.

We now apply the above result to several concrete cases. To this end let $C([0,1])$ denote the continuous functions on $[0,1]$, let $L^{\infty}([0,1])$ denote the set of equivalence classes of essentially bounded Lebesgue measurable functions and regard $C([0,1]) \subseteq L^{\infty}([0,1])$ by identifying a continuous function with its equivalence class. Since $L^{\infty}([0,1])$ is an injective von Neumann algebra, we have copies of $I(C([0,1]))$ embedded completely order isomorphically inside $L^{\infty}([0,1])$ and corresponding minimal (completely) positive projections onto these copies of the injective envelope. As before, we will have that the intersection of the ranges of all these projections is exactly the set of all elements of $L^{\infty}([0,1])$ that are fixed by every positive map that fixes $C([0,1])$. We let $\mathcal{F}(C([0,1]))$ denote this space.

To understand this example, it helps to notice some facts about sup's and inf's in $L^{\infty}([0,1])$. Letting $[g] \in L^{\infty}([0,1])$, recall that the set of points $x$ such that

$$
\lim _{h \rightarrow 0^{+}} \frac{1}{2 h} \int_{x-h}^{x+h} g(t) d m(t)
$$


exists is independent of the particular choice of function from the equivalence class of $g$ and is a set of full measure. These points are called the Lebesgue points of $g$. We let $E_{g}$ denote the set of Lebesgue points of $g$ and we let $\tilde{g}$ be the function whose domain is $E_{g}$ and which is equal to this limit at each Lebesgue point.

Given $[g] \in L^{\infty}([0,1])_{h}$, we define

$$
g_{l}(t)=\sup \left\{f(t): f \in C([0,1])_{h}, f(x) \leq \tilde{g}(x) \forall x \in E_{g}\right\}
$$

and

$$
g_{u}(t)=\inf \left\{f(t): f \in C([0,1])_{h}, g(x) \leq f(x) \forall x \in E_{g}\right\} .
$$

Note that $g_{l}$ is lower semicontinuous, $g_{u}$ is upper semicontinuous and $g_{l}(x) \leq$ $\tilde{g}(x) \leq g_{u}(x), \forall x \in E_{g}$. We should also note that these functions are not the usual upper and lower envelopes of $g$ that one encounters in Riemann integration. The usual lower and upper envelopes, which we will denote $g^{l}$ and $g^{u}$, are defined as above with $g$ in the place of $\tilde{g}$ and the inequalities required to hold at all points. For example, if $g$ is the characteristic function of the rationals, then $g^{l}$ is constantly 0 while $g^{u}$ is constantly 1 , but $E_{g}=[0,1]$ and $g_{l}(x)=\tilde{g}(x)=g_{u}(x)=0$. Note that for $x \in E_{g}$, we have that $f^{l}(x) \leq f_{l}(x) \leq \tilde{g}(x) \leq g_{u}(x) \leq g^{u}(x)$.

Proposition 4.26. Let $[g] \in L^{\infty}([0,1])_{h}$. Then in the lattice of $L^{\infty}([0,1])_{h}$ we have that

$$
\ell_{C([0,1])}([g])=\sup \left\{[f]: f \in C([0,1])_{h},[f] \leq[g]\right\}=\left[g_{l}\right]
$$

and

$$
u_{C([0,1])}([g])=\inf \left\{[f]: f \in C([0,1])_{h},[g] \leq[f]\right\}=\left[g_{u}\right] .
$$

Proof. We only prove the first equality. Let $[h]$ denote the supremum. If $f \in$ $C([0,1])_{h}$ and $[f] \leq[g]$, then $[f] \leq[h]$ and so $f \leq g$, a.e. $m$ and $f \leq h$, a.e. $m$. Hence, $f(x) \leq \tilde{g}(x), \forall x \in E_{g}$ and so $f(x) \leq g_{l}(x), \forall x \in E_{g}$. Since this set is full measure, $[f] \leq\left[g_{l}\right]$ and so $[h] \leq\left[g_{l}\right]$. But, we also have that $f(x) \leq \tilde{h}(x), \forall x \in E_{h}$. Hence, $g_{l}(x) \leq \tilde{h}(x), \forall x \in E_{h} \cap E_{g}$ and so, $\left[g_{l}\right] \leq[h]$, since $E_{h} \cap E_{g}$ is a set of full measure.

Note that unlike the case of the continuous functions, it is possible for two Riemann integrable functions to be equal almost everywhere without being equal. Thus, the inclusion of the Riemann integrable functions into $L^{\infty}([0,1])$ is not a monomorphism. Moreover, if a function is equal almost everywhere to a Riemann integrable function, it need not be Riemann integrable.

Theorem 4.27. For $C([0,1]) \subset L^{\infty}([0,1])$, the set $\mathcal{F}_{L^{\infty}([0,1])}(C([0,1]))$ is equal to the set of equivalence classes of Riemann integrable functions. That is, $[g] \in$ $\mathcal{F}_{L^{\infty}([0,1])}(C([0,1]))$ if and only if $f=g$, a.e. for some Riemann integrable function $f$.

Proof. By the above theorem, we have that $[g] \in \mathcal{F}_{L^{\infty}([0,1])}(C([0,1]))_{h}$ if and only if $\left[g_{l}\right]=[g]=\left[g_{u}\right]$. Since $g_{l}(x) \leq g_{u}(x), \forall x \in[0,1]$ and are equal almost everywhere, these functions are both Riemann integrable. Thus, if $[g] \in \mathcal{F}_{L^{\infty}([0,1])}(C([0,1]))_{h}$, then $g=g_{l}$ a.e. and $g_{l}$ is Riemann integrable.

Conversely, if $g=f$ a.e. are real-valued and $f$ is Riemann integrable, then $f^{l}=f^{u}$ a.e. and hence from the above inequalities, $f_{l}=f_{u}$ a.e., so that $\left[g_{l}\right]=$ $\left[f_{l}\right]=[f]=[g]=\left[f_{u}\right]=\left[g_{u}\right]$, which implies that $[g] \in \mathcal{F}_{L^{\infty}([0,1])}(C([0,1]))$. 
Corollary 4.28. Let $\pi: C([0,1]) \rightarrow B\left(L^{2}([0,1])\right)$ be the ${ }^{*}$-monomorphism given by $\pi(f)=M_{f}$, where $M_{f}$ denotes the operator of multiplication by $f$. Then $\mathcal{F}(\pi(C([0,1])))=\left\{M_{f}: f \in \mathcal{R}([0,1])\right\}$, where $\mathcal{R}([0,1])$ denotes the set of Riemann integrable functions and hence is a $C^{*}$-algebra.

Proof. We have that $\pi(C([0,1]))^{\prime}=\pi(C([0,1]))^{\prime \prime}=\left\{M_{f}: f \in L^{\infty}([0,1])\right\} \equiv$ $L^{\infty}([0,1])$ and the result follows.

The last statement follows from the fact that the Riemann integrable functions are a $C^{*}$-algebra and that $g \rightarrow M_{g}$ is a ${ }^{*}$-homomorphism.

We now consider a discrete case. Let $X$ be a compact, Hausdorff space, let $\ell^{\infty}(X)$ denote the bounded functions on $X$ and let $L S C(X)$ and $U S C(X)$ denote the real-valued lower semicontinuous and upper semicontinuous functions on $X$, respectively. Also, let $\left\{x_{i}\right\}_{i \in I}$ be a dense set in $X$. Recall that a function is lower semicontinuous (respectively, upper semicontinuous) if and only if it is the supremum (respectively, infimum) of the continuous real-valued functions that are less (respectively, greater) than it.

Proposition 4.29. Let $\pi: \ell^{\infty}(X) \rightarrow \ell^{\infty}(I)$ be defined by $\pi(f)(i)=f\left(x_{i}\right)$. Then

$$
\mathcal{F}_{\ell^{\infty}(I)}(\pi(C(X)))_{h}=\pi(L S C(X)) \cap \pi(U S C(X)) .
$$

Proof. We have that $h \in \mathcal{F}_{\ell^{\infty}(I)}(\pi(C(X)))_{h}$ if and only if $h=\sup \{\pi(f): f \in$ $\left.C(X)_{h}, \pi(f) \leq h\right\}=\inf \left\{\pi(f): f \in C(X)_{h}, h \leq \pi(f)\right\}$.

Let $g_{l}(x)=\sup \left\{f(x): f \in C(X)_{h}, \pi(f) \leq h\right\}$ and $g_{u}(x)=\inf \{f(x): f \in$ $\left.C(X)_{h}, \pi(f) \leq h\right\}$. Then $g_{l}$ is lower semicontinuous and $g_{u}$ is upper semicontinuous and $h=\pi\left(g_{l}\right)=\pi\left(g_{u}\right)$. Hence, $\mathcal{F}_{\ell^{\infty}(I)}(\pi(C(X)))_{h} \subseteq \pi(L S C(X)) \cap \pi(U S C(X))$.

Conversely, let $h \in \pi(L S C(X)) \cap \pi(U S C(X))$, say $h=\pi\left(g_{l}\right)=\pi\left(g_{u}\right)$. Note that for $f \in C(X)_{h}$, we have that $\pi(f) \leq h$ if and only if $f \leq g_{l}$. Hence, $\sup \{\pi(f)$ : $\left.f \in C(X)_{h}, \pi(f) \leq h\right\}=\sup \left\{\pi(f): f \in C(X)_{h}, f \leq g_{l}\right\}=\pi\left(g_{l}\right)=h$. Similarly, $h=\inf \left\{\pi(f): f \in C(X)_{h}, h \leq \pi(f)\right\}$ and the result follows.

Corollary 4.30. Let $X$ be a compact, Hausdorff space, let $\left\{x_{i}\right\}_{i \in I}$ be a dense set of distinct points in $X$, let $\left\{e_{i}\right\}$ denote the canonical orthonormal basis for $\ell^{2}(I)$, and let $\pi: \ell^{\infty}(X) \rightarrow B\left(\ell^{2}(I)\right)$ be the diagonal representation defined by $\pi(f) e_{i}=f\left(x_{i}\right) e_{i}, \forall i$. Then $\mathcal{F}(\pi(C(X)))_{h}=\pi(L S C(X)) \cap \pi(U S C(X))$.

Proof. The result follows as above, since $\pi(C(X))^{\prime \prime}=\pi(C(X))^{\prime}$.

Consider the case of $X=[0,1]$, with a dense subset given by an enumeration of the rationals, $\left\{r_{n}\right\}_{n \in \mathbb{N}}$, and $\pi: \ell^{\infty}([0,1]) \rightarrow B\left(\ell^{2}(\mathbb{N})\right)$, given by the above formula. If we consider an interval with irrational endpoints, $a$ and $b$, then $\pi\left(\chi_{[a, b]}\right)=\pi\left(\chi_{(a, b)}\right)$ and so this projection belongs to $\mathcal{F}(\pi(C([0,1])))$. However, it can be seen that no finite rank diagonal projection or a projection corresponding to an interval with a rational endpoint belongs to $\mathcal{F}(\pi(C([0,1])))$.

\section{ACKNowledgments}

The author wishes to thank Ken Davidson, Roger Smith and Ivan Todorov for various conservations that contributed to this work. 


\section{REFERENCES}

[1] W. B. Arveson, An Invitation to $C^{*}$-algebras, Graduate Texts in Mathematics, vol. 39, Springer-Verlag, New York, 1976. MR0512360 (58:23621)

[2] B. E. Blackadar, Weak Expectations and Nuclear $C^{*}$-algebras, Indiana University Mathematics J., Vol. 27, No. 6(1978), 1021-1026. MR511256 (80d:46110)

[3] D. P. Blecher and C. LeMerdy, Operator Algebras and Their Modules, London Mathematical Society Monographs, vol. 30, Oxford Science Publications, Oxford, England, 2004. MR2111973 (2006a:46070)

[4] M. D. Choi, A Schwarz inequality for positive linear maps on $C^{*}$-algebras, Illinois J. Math. 18(1974), 565-574. MR0355615 (50:8089)

[5] U. Haagerup, Decompositions of completely bounded maps on operator algebras, unpublished manuscript, Sept. 1980.

[6] M. Hamana, Injective envelopes of $C^{*}$-algebras, J. Math. Soc. Japan, Vol. 31, No. 1, (1979), 181-197. MR519044 (80g:46048)

[7] M. Hamana, Injective envelopes of operator systems, Publ. Res. Inst. Math. Sci. 15(1979), no. 3, 773-785. MR.566081 (81h:46071)

[8] M. Hamana, Regular embeddings of $C^{*}$-algebras in monotone complete $C^{*}$-algebras, J. Math. Soc. Japan 33(1981), no. 1, 159-183. MR597486 (82i:46089)

[9] R. V. Kadison and J. R. Ringrose, Fundamentals of the theory of operator algebras Vol. 1: Elemental Theory, Academic Press, New York (1983). The current publication is from Amer. Math. Soc., Providence, RI, 1997, with the same title and contents.

[10] G. Pisier, Introduction to Operator Space Theory, London Mathematical Society Lecture Note Series 294, Cambridge University Press, Cambridge, United Kingdom, 2003. MR2006539 (2004k:46097)

[11] Z.-J. Ruan, Injectivity of Operator Spaces, Trans. Amer. Math. Soc. 315(1989), 89-104. MR.929239 (91d:46078)

[12] R. R. Smith, Completely bounded module maps and the Haagerup tensor product, J. Funct. Anal. 102(1991), no. 1, 156-175. MR.1138841 (93a:46115)

[13] J. D. Maitland Wright, Every monotone $\sigma$-complete $C^{*}$-algebra is the quotient of its Baire* envelope by a two-sided $\sigma$-ideal, J. London Math. Soc. (2), 6(1973), 210-214. MR0318903 (47:7449)

[14] J. D. Maitland Wright, On minimal $\sigma$-completions of $C^{*}$-algebras, Bull. London Math. Soc., 6(1974), 168-174. MR0370208 (51:6437)

[15] J. D. Maitland Wright, Regular $\sigma$-completions of $C^{*}$-algebras, J. London Math. Soc. (2), 12(1976), 299-309. MR0397428 (53:1287)

Department of Mathematics, University of Houston, Houston, Texas 77204-3476

E-mail address: vern@math.uh.edu 